\title{
Importance of the fluid-particle drag model in predicting segregation in bidisperse gas-solid flow
}

\author{
Mohammad Mehrabadi ${ }^{\mathrm{a}, \mathrm{b}}$, Sudheer Tenneti ${ }^{\mathrm{a}, \mathrm{c}}$, Shankar Subramaniam ${ }^{\mathrm{a}, *}$ \\ ${ }^{a}$ Center for Multiphase Flow Research, Department of Mechanical Engineering, \\ Iowa State University, Ames, IA 50011, USA \\ ${ }^{b}$ Department of Aerospace Engineering, University of Illinois \\ at Urbana-Champaign, IL 61801 USA \\ ${ }^{c}$ CD-Adapco, Lebanon, NH, 03766, USA
}

\section{Abstract}

The slip velocity between particle size classes in a homogeneous bidisperse gas-solid flow is quantified using particle-resolved direct numerical simulation (PR-DNS). This slip velocity is the key characteristic of size segregation in industrial devices. The ability of current gas-particle drag models to predict this slip velocity is examined by simultaneously solving the mean momentum equations for the gas phase and dispersed phases. PR-DNS of fixed particle assemblies is then used to validate and improve the bidisperse gas-particle drag model. The drag model inferred from bidisperse fixed beds is compared with the drag force measured from PR-DNS of freely evolving bidisperse suspensions. The ability of this new model to predict the slip velocity between particle size classes in a bidisperse gas-solid flow is also examined.

Keywords: Particle-resolved DNS, Bidisperse suspension, Gas-solid flow, Segregation, Drag law

\footnotetext{
*Corresponding author

Email address: shankar@iastate.edu (Shankar Subramaniam)
} 


\section{Introduction}

Gas-solid flows are commonly found in industrial applications such as fluidized-bed combustion, fluid catalytic cracking, coal gasification, and biomass energy generation (Fan et al., 2004). In these flows, the dispersed phase is generally polydisperse, meaning that there is a distribution in the particle size and density. These distributions lead to a complex interplay of various gas-particle and particle-particle interactions that in turn give rise to particle mass flux of one particle class with respect to the others (Holloway et al., 2011). This mass flux is the key signature of the segregation phenomenon observed in gas-solid suspensions (Nienow et al., 1987; Wu and Baeyens, 1998; Goldschmidt et al., 2003; Bokkers et al., 2004; Chew et al., 2011; Hoffmann et al., 1993; Fan and Fox, 2008; Holloway et al., 2011; Norouzi et al., 2012). Segregation is desirable in processes where separation of particle classes is required, while it may not be beneficial in processes where a homogeneous mixture of the dispersed phase is needed. Therefore, accurate modeling of the size and density-dependent particle mass flux in polydisperse suspensions sheds light on the segregation phenomenon and provides useful information for better engineering design.

In the gas-solid flow applications mentioned above, the material density ratio ranges from $\rho^{(p)} / \rho^{(f)} \sim \mathcal{O}(100)$ to $\mathcal{O}(1000)$ and the particle diameter varies from $d_{p} \sim 50 \mu \mathrm{m}$ to $500 \mu \mathrm{m}$. The interaction of the gas phase with classes of particles in the form of gas-particle drag force depends on particle material density as well as particle diameter. This dependence leads to a net mean hydrodynamic force on each particle class that results in relative mean motion between particle classes. On the other hand, the hydrodynamic forces 
acting on each particle give rise to the growth of particle velocity fluctuations characterized by particle granular temperature (Tenneti et al., 2010). The random motion of particles increases the probability of a particle colliding either with other particles of the same class, or with particles belonging to other classes. Although particle collisions in a monodisperse system do not result in a net change of mean momentum, in a polydisperse system there is a transfer of mean momentum from one particle class to another even if the collisions are elastic. This is referred to as the particle-particle drag force. This particle-particle drag hinders the increase of mean slip velocity between particle classes, and thus reduces segregation.

In a polydisperse suspension, the balance between gas-particle and particleparticle interactions governs the relative motion of particle classes. Bidisperse flows with two particle classes of differing size or density (or both size and density) are the simplest polydisperse flow. The interaction of two particle classes in a bidisperse flow also forms the basis for models of polydisperse flow that rely on a pairwise additive interaction between particle classes (Syamlal et al., 1993). Therefore, this work focuses on bidisperse flows.

The study of segregation in practical applications of polydisperse gassolid flow is complicated by wall effects and statistical inhomogeneity, i.e. mean flow quantities such as average fluid velocity vary with spatial location. Therefore, it is difficult to isolate the effect of individual terms such as fluidparticle drag in such problems. In a statistically homogeneous flow the mean flow quantities are spatially uniform and wall effects are absent, which allows for easy evaluation of models for unclosed terms such as the fluid-particle drag, and their subsequent development. Therefore, in this work we focus on 
statistically homogeneous bidisperse gas-solid flow.

Although the kinetic theory of bidisperse granular flow predicts that nonzero particle mass flux arises only if there are gradients in particle number density, particle granular temperature, or external forces (Garzó et al., 2007), the interaction of particles with the ambient fluid provides another mechanism for segregation in gas-solid flow. At this point there is no comprehensive mapping of the regions in parameter space defined by particle class ratios of density, size and volume fraction $\left(\rho_{2} / \rho_{1}, d_{2} / d_{1}, \phi_{2} / \phi_{1}\right)$ which shows where segregation or mixing are expected.

Experimental investigations provide useful information about gas-solid flows (Bokkers et al., 2004; Chew and Hrenya, 2011) but experiments include wall effects and it is difficult to isolate the effects of individual terms in these studies. Furthermore, limited optical access into fluidized beds limits the applicability of experimental investigations to either dilute suspensions (Lee and Durst, 1982; Rogers and Eaton, 1991; Sato et al., 1996; Oakley et al., 1997; Kiger and Pan, 2000) or pseudo two-dimensional experimental setup (Goldschmidt et al., 2003; Bokkers et al., 2004).

Particle-resolved direct numerical simulation (PR-DNS) is an alternative approach that is well suited for discovering flow physics in particle-laden flow. The PR-DNS approach not only provides detailed information about the hydrodynamic velocity and pressure fields, it also provides particles trajectories along with their velocities and accelerations. Furthermore, properly designed PR-DNS studies can be used to either propose closure models used in CFD calculations of gas-solid flow, or to test the validity of these closure models.

The PR-DNS methodology has been successfully used to propose clo- 
sure models for the average interphase momentum transfer, whose principal contribution is the average gas-solid drag. Early studies focused on monodisperse gas-solid flows and they are important because the bidisperse drag law is often proposed as a modification of the monodisperse drag law. Hill et al. (2001a) used the Lattice Boltzmann Method (LBM) to propose a drag model for monodisperse arrays of ordered and randomly distributed particles in the Stokes flow regime, and validated their results for low solid volume fraction with theoretical analysis. Hill et al. (2001b) extended their drag model to moderate Reynolds numbers. In a similar attempt, van der Hoef et al. (2005) also used LBM to a propose drag model for monodisperse gas-solid flow in the Stokes regime using fixed particle assemblies. Beetstra et al. (2007) used LBM to solve for flow past fixed particle assemblies to incorporate the effect of higher mean slip Reynolds number (up to 1000) into the low-Reynoldsnumber monodisperse drag model of van der Hoef et al. (2005). Tenneti et al. (2011) performed PR-DNS based on the Particle-resolved Reconcilable Immersed Boundary Method (PUReIBM) to solve the Navier-Stokes equations and reported the drag force in gas-solid flows using fixed particle assemblies over a wide range of flow parameters $\left(0.1 \leq \phi \leq 0.5\right.$ and $0.01 \leq R e_{m} \leq 300$. They also proposed a drag law as a function of the solid-phase volume fraction $\phi$ and mean slip Reynolds number $R e_{m}$ (for the definition of the Reynolds number see Eq. 5).

In an attempt to generalize the quantification of interphase momentum transfer to polydisperse systems, van der Hoef et al. (2005) performed PRDNS of bidisperse gas-solid flow in the low Reynolds number regime. They proposed a bidisperse drag law that expresses the drag force experienced by 
each particle size class in terms of the drag experienced by an equivalent monodisperse suspension multiplied by a function of the particle size ratio. This function of the particle size ratio is referred to as VDH bidisperse drag model from hereon. The maximum particle size ratio of the bidisperse configurations used in their study was $1: 4$. Sarkar et al. (2009) then confirmed the applicability of VDH drag law even for extreme particle size ratios up to 1 : 10. Yin and coworkers (Yin and Sundaresan, 2009; Holloway et al., 2010) reasoned that the relative motion of particles in a suspension indirectly affects the hydrodynamic force experienced by neighboring particles through the lubrication force. They used frozen particle configurations in the Stokes flow regime in their PR-DNS setup with assigned mean velocity to each particle class, which gives rise to evolution of flow motion through implementation of the no-slip and no-penetration boundary condition at particle surface. This methodology enabled them to explore the effect of indirect particle-particle interaction on the mean drag, and propose a new model for polydisperse suspensions in the Stokes flow regime. However, Tenneti et al. (2011) showed that this methodology is not Galilean-invariant, and may incur significant error in moderate Reynolds number flows. Rong et al. (2014) also used LBM to propose a drag model for the hydrodynamic force on each particle size class and is expressed in terms of a monodisperse drag law (Rong et al., 2013).

All of the aforementioned bidisperse PR-DNS exploited fixed or frozen particle assemblies as an approximation to real gas-solid suspensions with freely moving particles. In fixed particle assemblies, it is assumed that all particles have the same velocity. This assumption is useful for calculating the 
gas-particle drag in both monodisperse and polydisperse configurations, and is relatively inexpensive for parametric studies. Nevertheless, it is expected that the differences in hydrodynamic and collisional forces on particle classes result in the development of different mean velocities for each particle class. The significance of hydrodynamic and collisional forces on the development of the mean velocity difference between particle classes in freely evolving suspensions has not been reported, to the best of our knowledge.

Although the drag model proposed by van der Hoef and coworkers (van der Hoef et al., 2005; Beetstra et al., 2007) solely depends on particle size ratio, models of Syamlal and O'Brien (1987), Yin and Sundaresan (2009), and Holloway et al. (2010) also consider the mean slip velocity between particle classes. It is not known a priori if the particle size ratio alone is adequate to characterize the mean gas-particle drag, and how well this holds in freely evolving suspensions.

In the current study we study the influence of the gas-particle drag model on the evolution of the mean slip velocity between two particle size classes in a bidisperse gas-solid flow. We start by deriving the mathematical description of a homogeneous bidisperse gas-solid flow in Section 2. We then provide details of our numerical approach to simulate gas-solid suspensions in Section 3. In Section 4, we perform PR-DNS of a homogeneous bidisperse freely evolving gas-solid suspension at a moderate Reynolds number to measure the mean slip velocity between the two particle size classes that gives rise to particle mass flux. We also assess the ability of gas-particle drag models that are widely used in two-fluid model (TFM) CFD simulations to predict the slip velocity between the two particle size classes in Section 5 . 
Also in an attempt to improve current bidisperse drag models, we report results from highly resolved PR-DNS of homogeneous bidisperse fixed particle assemblies and quantify the drag force on each particle size class in Section 6. We also discuss the remaining challenges in developing an accurate bidisperse gas-particle drag model, and possible directions for further improvement in Section 7. Finally, conclusions are summarized in Section 8.

\section{Homogeneous bidisperse gas-solid suspension}

Statistical representations of multiphase flow are widely used in macroscale descriptions based on the averaged conservation equations of mass, momentum, and energy (see Appendix A for more detail) that are used in devicescale computations. These averaged equations include unclosed terms that arise due to the statistical averaging procedure, and require modeling. A better understanding of the physics governing the microscale (at the scale of individual particles) and mesoscale (scale of clusters of hundreds of particles) dynamics of multiphase flow is needed for accurately modeling these unclosed terms. The PR-DNS approach is currently used to study the microscale dynamics because of the high computational cost involved in extending it to the mesoscale. Studying the microscale dynamics of gas-solid flows in canonical problems enables us to isolate the effect of specific unclosed terms, and thus quantify and characterize their behavior. For instance, in homogeneous gassolid suspensions we can quantify the contribution of gas-particle drag and

particle-particle drag to the mean momentum equations in the absence of transport terms, and thus improve the predictive capabilities of drag models in representing these unclosed terms. 
In light of the homogeneity assumption all spatial variations of mean quantities disappear. Therefore, the conservation equation of mass (Eq. A.2) is trivially satisfied. In addition, in the conservation of mean momentum equation (Eq. A.3), the transport of second moment terms, such as the Reynolds stress tensor, and also the contribution from the divergence of viscous stresses disappear. Thus, the mean momentum equation reduces to a balance between the rate of change of the mean momentum, mean pressure gradient (or other types of body force), mean interphase momentum transfer due to hydrodynamic and collisional interactions, which are presented in more detail in the following sub-section.

\subsection{Mean momentum equations}

The mean momentum equations of the fluid phase and a polydisperse solid phase are given in Appendix A. For homogeneous bidisperse suspensions with constant volume fractions and densities and in the absence of gravity, these equations simplify to:

$$
\rho^{(f)} \phi^{(f)} \frac{\partial}{\partial t}\left\langle\mathbf{u}^{(f)}\right\rangle=-\phi^{(f)}\langle\mathbf{g}\rangle-\sum_{\alpha=1}^{2} \underbrace{\left\langle\boldsymbol{\tau} \cdot \mathbf{n}^{(\alpha)} \delta\left(\mathbf{x}-\mathbf{x}_{\alpha}^{(I)}\right)\right\rangle}_{\left\langle\mathbf{s}_{\mathrm{h}}^{(\alpha)}\right\rangle},
$$

and

$$
\rho^{(\alpha)} \phi^{(\alpha)} \frac{\partial}{\partial t}\left\langle\mathbf{u}^{(\alpha)}\right\rangle=-\phi^{(\alpha)}\langle\mathbf{g}\rangle+\underbrace{\left\langle\boldsymbol{\tau} \cdot \mathbf{n}^{(\alpha)} \delta\left(\mathbf{x}-\mathbf{x}_{\alpha}^{(I)}\right)\right\rangle}_{\left\langle\mathbf{s}_{\mathrm{h}}^{(\alpha)}\right\rangle}+\left\langle\mathbf{f}_{\mathrm{coll}}^{(\beta \rightarrow \alpha)}\right\rangle,
$$

where $\rho^{(f)}$ is the fluid-phase density, $\phi^{(f)}$ is the corresponding volume fraction, $\left\langle\mathbf{u}^{(f)}\right\rangle$ is the mean fluid velocity, $\langle\mathbf{g}\rangle$ is the mean pressure gradient, $\boldsymbol{\tau}$ is the stress tensor at the fluid-solid interface that includes the fluctuating pressure 
and the viscous stress, $\mathbf{n}^{(\alpha)}$ is the normal vector at the surface of particles belonging to $\alpha^{\text {th }}$ class pointing into the fluid phase, $\delta\left(\mathbf{x}-\mathbf{x}_{\alpha}^{(I)}\right)$ is a generalized delta function at the fluid-particle interface $\mathbf{x}_{\alpha}^{(I)}$, and $N_{c}$ is the number of particle classes. Similarly, $\rho^{(\alpha)}$ is the $\alpha^{\text {th }}$ particle class density, $\phi^{(\alpha)}$ is the corresponding volume fraction, $\left\langle\mathbf{u}^{(\alpha)}\right\rangle$ is the particle class mean velocity, and $\left\langle\mathbf{f}_{\text {coll }}^{(\beta \rightarrow \alpha)}\right\rangle$ in Eq. 2 is the mean momentum transfer between particle classes $\alpha$ and $\beta$, identified as the particle-particle drag. The term $\left\langle\mathbf{s}_{\mathrm{h}}^{(\alpha)}\right\rangle$ represents the transfer of mean momentum to the solid phase due to the hydrodynamic forces on particle class $\alpha$. In gas-solid flows the hydrodynamic force is modeled as the drag force since that is the principal contribution, although in shear flows and with rotating particles (Kurose and Komori, 1999; Bagchi and Balachandar, 2002) the lift force can also be important. The sum of all hydrodynamic forces on the right-hand side of Eq. 1 represents the total hydrodynamic force experienced by solid particles, denoted by:

$$
\left\langle\mathbf{s}_{\mathrm{h}}\right\rangle=\sum_{\alpha=1}^{2}\left\langle\mathbf{s}_{\mathrm{h}}^{(\alpha)}\right\rangle .
$$

Again this is modeled as the average gas-particle drag, and the average gasparticle and particle-particle drag forces are not known a priori and need to be modeled in two-fluid model simulations.

\subsection{Slip velocity between the two particle classes}

It is worthwhile to examine the mean momentum equation governing the slip velocity between the two particle classes since this slip velocity determines the average particle mass flux. The slip velocity between the two particle classes $\left\langle\mathbf{W}^{(\beta, \alpha)}\right\rangle$ in a bidisperse suspension in the absence of gravity and driven by a mean pressure gradient is obtained by subtracting the 
conservation equation of mean momentum corresponding to particle class $\alpha$ from that of particle class $\beta$ in Eq. 2, resulting in

$$
\begin{aligned}
\frac{d}{d t}\left\langle\mathbf{W}^{(\beta, \alpha)}\right\rangle= & -\langle\mathbf{g}\rangle\left(\frac{1}{\rho^{(\beta)}}-\frac{1}{\rho^{(\alpha)}}\right) \\
& +\left(\frac{1}{\rho^{(\beta)} \phi^{(\beta)}}\left\langle\mathbf{s}_{\mathrm{h}}^{(\beta)}\right\rangle-\frac{1}{\rho^{(\alpha)} \phi^{(\alpha)}}\left\langle\mathbf{s}_{\mathrm{h}}^{(\alpha)}\right\rangle\right) \\
& -\left\langle\mathbf{f}_{\text {coll }}^{(\beta \rightarrow \alpha)}\right\rangle\left(\frac{1}{\rho^{(\alpha)} \phi^{(\alpha)}}+\frac{1}{\rho^{(\beta)} \phi^{(\beta)}}\right)
\end{aligned}
$$

Note that conservation of linear momentum for collisions between a pair of particles necessitates that the collisional force between two particles be equal but opposite. Therefore, the mean collisional forces between two particle size classes is conservative, that is $\left\langle\mathbf{f}_{\text {coll }}^{(\alpha \rightarrow \beta)}\right\rangle=-\left\langle\mathbf{f}_{\text {coll }}^{(\beta \rightarrow \alpha)}\right\rangle$. Equation 4 for the evolution of the mean slip velocity between particle classes shows that both gas-particle drag and particle-particle drag forces contribute to the rate of change of $\left\langle\mathbf{W}^{(\beta, \alpha)}\right\rangle$ that leads to particle mass flux in a homogeneous bidisperse suspension. Therefore, accurate modeling of both contributions is essential in particle-laden flow analysis.

\subsection{Characterization of bidisperse gas-solid suspensions}

The dynamics of a bidisperse gas-solid suspension depends on the regime of the flow in a high-dimensional parameter space that is characterized by:

1. total solid-phase volume fraction $\phi^{(p)}$

2. ratio of particle class volume fraction to the total solid-phase volume fraction $x_{\alpha}=\phi^{(\alpha)} / \phi^{(p)}$

3. ratio of particle class density to fluid-phase density $\rho^{(\alpha)} / \rho^{(f)}$

4. ratio of particle class diameter to Sauter mean diameter $y_{\alpha}=d_{\alpha} /\langle d\rangle$ 
5. mean slip Reynolds number $R e_{m}$, and

6. particle class Stokes number $S t_{\alpha}$.

The mean slip Reynolds number based on the Sauter mean diameter $\langle d\rangle=$ $\left(\sum_{\alpha=1}^{N_{c}} \phi^{(\alpha)} /\left(\phi d_{\alpha}\right)\right)^{-1}$ is defined as

$$
R e_{m}=\frac{\phi^{(f)}|\langle\mathbf{W}\rangle|\langle d\rangle}{\nu^{(f)}}
$$

where the mixture mean slip velocity is defined as the difference between the mass-weighted solid-phase velocity (see Eq. B.7) and the fluid-phase velocity, i.e.,

$$
\langle\mathbf{W}\rangle=\left\langle\widetilde{\mathbf{u}^{(p)}}\right\rangle-\left\langle\mathbf{u}^{(f)}\right\rangle .
$$

The particle class Stokes number is defined as the particle response time to the characteristic fluid flow time scale that is given by

$$
S t_{\alpha}=\frac{\tau^{(\alpha)}}{\tau^{(f)}}=\frac{1}{18} \frac{\rho^{(\alpha)}}{\rho^{(f)}}\left(\frac{d_{\alpha}}{\langle d\rangle}\right)^{2} R e_{m} .
$$

These parameters are used to characterize the gas-solid flows considered in this study.

\section{Numerical method}

Several numerical methods have been developed for particle-resolved direct numerical simulation (PR-DNS) of fluid-solid flows, such as the finite element approach of Johnson and Tezduyar (1997), the immersed boundary method of Peskin (2002), the Lattice-Boltzmann method used by Chen and Doolen (1998), a combination of the two former methods implemented by Feng and Michaelides (2004), and the PHYSALIS method of Prosperetti 
and Oguz (2001). The Lattice-Boltzmann based PR-DNS solvers have been extensively used to study bidisperse gas-solid flows (van der Hoef et al., 2005; Beetstra et al., 2007; Sarkar et al., 2009; Yin and Sundaresan, 2009; Holloway et al., 2010). In this study, we use the particle-resolved uncontaminated-fluid reconcilable immersed boundary method (PUReIBM) of Tenneti et al. (2010). The salient feature of PUReIBM is that the IB forcing in PUReIBM is nonzero only inside the solid phase, and the fluid-phase is uncontaminated by the IB forcing. Therefore, the velocity and pressure in the fluid phase satisfy the unmodified Navier-Stokes equations. In addition, the hydrodynamic force experienced by a particle is computed directly from the stress tensor at the particle surface that is obtained from this uncontaminated fluid flow solution. This feature enables us to directly compare the PR-DNS solution with any random-field theory of multiphase flow (Garg et al., 2010; Tenneti et al., 2011), and in particular we can quantify the slip velocity between the two size classes $\left\langle\mathbf{W}^{(\beta, \alpha)}\right\rangle$ in Eq. 4.

This method is shown to be accurate and numerically convergent (Garg et al., 2010; Tenneti et al., 2011). In addition, PUReIBM has been successfully used to simulate fixed particle assemblies (Tenneti et al., 2010, 2011; Sun et al., 2015) and freely evolving suspensions of monodisperse gas-solid flows (Subramaniam et al., 2014; Mehrabadi et al., 2015; Tenneti et al., 2016). The extension of the PUReIBM formulation to account for polydisperse gassolid suspensions is straightforward (see Appendix B).

In PUReIBM, the three dimensional Navier-Stokes equations are solved on a Cartesian grid for the whole computational domain in an accelerating frame that moves at the mean velocity of the particles. The instantaneous 
conservation equations of mass and momentum are

$$
\nabla \cdot \mathbf{u}=0
$$

and

$$
\rho^{(f)} \frac{\partial \mathbf{u}}{\partial t}+\rho^{(f)} \mathbf{S}=-\mathbf{g}_{\text {IBM }}+\mu^{(f)} \nabla^{2} \mathbf{u}+\mathbf{f}-\rho^{(f)} \mathbf{A}_{f},
$$

respectively, where $\mathbf{u}$ is the instantaneous velocity, $\mathbf{S}=\nabla \cdot(\mathbf{u u})$ is the convective term in conservative form, $\mathbf{g}_{\mathrm{IBM}}=\nabla p$ is the pressure gradient, $\mathbf{f}$ is the immersed boundary (IB) forcing that accounts for the presence of particles, and $\mathbf{A}_{f}$ accounts for the acceleration of the frame of reference (Tenneti et al., 2010) that moves with the mean velocity of particles in freely evolving suspensions. This accelerating frame resembles a sedimentation problem with a gravitational acceleration that is different from standard gravity applied to each phase. This results in different net acceleration for the fluid phase and the solid phase, which gives rise to the appearance of a mean slip velocity between the two phases. The rate of change of the mean slip velocity is eventually balanced by the difference between the gas-particle drag force and the mean pressure gradient, appropriately scaled (Tenneti et al., 2010). Although both fluid phase and solid phase continually accelerate in the laboratory frame, the mean slip velocity between the two phases reaches a steady value. Numerical solution of the governing equations in the laboratory frame requires progressively smaller time steps as the mean velocities of the phases increase with time. However, in the accelerating frame of reference specified in Tenneti et al. (2010) the mean particle velocity is zero and all the velocities are bounded. Therefore, in this accelerating frame of reference the required time step does not progressively reduce, which enables 
us to continue simulations to any desired evolution time. More importantly, the simulation of freely evolving suspensions in an accelerating frame of reference (Tenneti et al., 2011) enables us to simulate suspensions at arbitrary Reynolds numbers while maintaining other parameters at fixed values. This is particularly relevant for drag model development to be used in multiphase CFD simulations because the gas-solid drag law is needed for arbitrary mean slip Reynolds number of the solid phase with respect to the fluid phase. This is in contrast to a gas-solid suspension in a gravitational field whose quasisteady mean Reynolds number based on the mean slip velocity between solid and fluid phases is uniquely determined by the particle to fluid density ratio and solid volume fraction. The case of gas-solid flow in a gravitational field is simply a special case of the entire suite of simulations that are possible using the accelerating frame setup. This results in the particles settling at a mean velocity corresponding to $R e_{m}$ in a quiescent fluid (not necessarily corresponding to the terminal velocity with $\left.g=9.81 \mathrm{~m} / \mathrm{s}^{2}\right)$.

In PUReIBM the particles are represented in a Lagrangian frame of reference at time $t$ by $\mathbf{X}^{(\alpha, i)}(t), \mathbf{V}^{(\alpha, i)}(t), \alpha=1$ and $2, i=1 \ldots N_{\alpha}$ with $\mathbf{X}^{(\alpha, n)}(t)$ and $\mathbf{V}^{(\alpha, n)}(t)$ being respectively the position and velocity of $i^{\text {th }}$ particle in the $\alpha^{\text {th }}$ size class. The position and translational velocity of each particle in 
class $\alpha$ evolve according to Newton's second law as

$$
\begin{aligned}
\frac{d \mathbf{X}^{(\alpha, i)}(t)}{d t} & =\mathbf{V}^{(\alpha, i)}(t), \\
m^{(\alpha, i)} \frac{d \mathbf{V}^{(\alpha, i)}(t)}{d t} & =-\left\langle\mathbf{g}_{\mathrm{IBM}}\right\rangle_{\mathcal{V}} V^{(\alpha, i)}+\mathbf{S}_{\mathrm{h}}^{(\alpha, i)}(t)+\sum_{\substack{j=1 \\
j \neq i}}^{N_{\alpha}} \mathbf{F}_{\text {coll }}^{(\alpha, j \rightarrow i)}(t) \\
& +\sum_{\substack{\beta=1 \\
\beta \neq \alpha}}^{2} \sum_{j=1}^{N_{\alpha}} \mathbf{F}_{\text {coll }}^{(\beta \rightarrow \alpha, j \rightarrow i)}(t)-m^{(\alpha, i)} \mathbf{A}_{f},
\end{aligned}
$$

where $m^{(\alpha, i)}$ and $V^{(\alpha, i)}$ are, respectively, the mass and volume of the $i^{\text {th }}$ particle of class $\alpha, \mathbf{S}_{\mathrm{h}}^{(\alpha, i)}$ is the hydrodynamic force acting on the $i^{\text {th }}$ particle with the form given in Eq. B.5, $\mathbf{F}_{\text {coll }}^{(\alpha, j \rightarrow i)}$ is the contact force on the $i^{\text {th }}$ particle from collisions with the $j^{\text {th }}$ particle in the same size class, and $\mathbf{F}_{\text {coll }}^{(\beta \rightarrow \alpha, j \rightarrow i)}$ is the contact force from collisions with the $k^{\text {th }}$ particle belonging to the size class $\beta^{\text {th }}$. Particle-particle interactions are treated using soft-sphere collisions based on a linear spring-dashpot contact mechanics model originally proposed by Cundall and Strack (1979). Further details of the particle-particle interaction are provided by Mehrabadi et al. (2015).

\section{PR-DNS of Freely Evolving Suspensions}

In this section, the PR-DNS of homogeneous bidisperse gas-solid flow considered in this study is presented. To generate a homogeneous particle configuration of a bidisperse suspension, non-overlapping spheres are first generated with particle centers on a cubic lattice. Particles are then assigned a Maxwellian velocity distribution and they undergo purely elastic collisions according to the soft-sphere model (Cundall and Strack, 1979) in the absence 
of the gas phase, establishing an equilibrium state with a homogeneous particle configuration (Tenneti et al., 2011). We consider two size class ratios with the details provided in Table 1. It should be noted that the particle size class diameter to Sauter mean diameter ratio is given by $y_{\alpha}=d_{\alpha} /\langle d\rangle$. We limit our analysis to a fixed density ratio for all particle classes, i.e. $\rho^{(1)}=\rho^{(2)}=\rho^{(p)}$, in order to only isolate the effect of particle size ratio. The solid-to-fluid density ratio is selected as $\rho^{(p)} / \rho^{(f)}=1000$. The mean slip Reynolds number is chosen to be $R e_{m}=50$ and the total solid-phase volume fraction is $\phi^{(p)}=0.3$. In order to achieve a desired mean slip Reynolds number at steady steady state, a specific mean pressure gradient is required. The formulation of PUReIBM in an accelerating frame for polydisperse systems (see Appendix B for further detail) enables us to easily attain this mean pressure gradient.

The length of the computational box for these simulations is chosen as $\mathcal{L}=6\langle d\rangle$, which is long enough for convergence of the drag force (Tenneti et al., 2011), and for the decorrelation of gas-phase velocity fluctuations that is needed when using periodic boundary conditions (Mehrabadi et al.,

Table 1: Details of bidisperse configurations for PR-DNS of freely evolving suspensions for the particle size class diameter to Sauter mean diameter ratio $y_{\alpha}=d_{\alpha} /\langle d\rangle$, size class volume fraction to total solid-phase volume fraction $\phi^{(\alpha)} / \phi^{(p)}$, number of particles in size classes $N_{\alpha}$, and grid resolution par particle for each size class $d_{\alpha} / \Delta_{x}$. In both cases, $\rho^{(p)} / \rho^{(f)}=1000, \phi^{(p)}=0.3$, and $R e_{m}=50$.

\begin{tabular}{lcccccccc}
\hline & $y_{1}$ & $y_{2}$ & $\phi^{(1)} / \phi^{(p)}$ & $\phi^{(2)} / \phi^{(p)}$ & $N_{1}$ & $N_{2}$ & $d_{1} / \Delta_{x}$ & $d_{2} / \Delta_{x}$ \\
\hline Case I & 0.75 & 1.125 & 0.25 & 0.75 & 73 & 65 & 23 & 34 \\
Case II & 0.5 & 1.5 & 0.25 & 0.75 & 247 & 27 & 15 & 45 \\
\hline
\end{tabular}




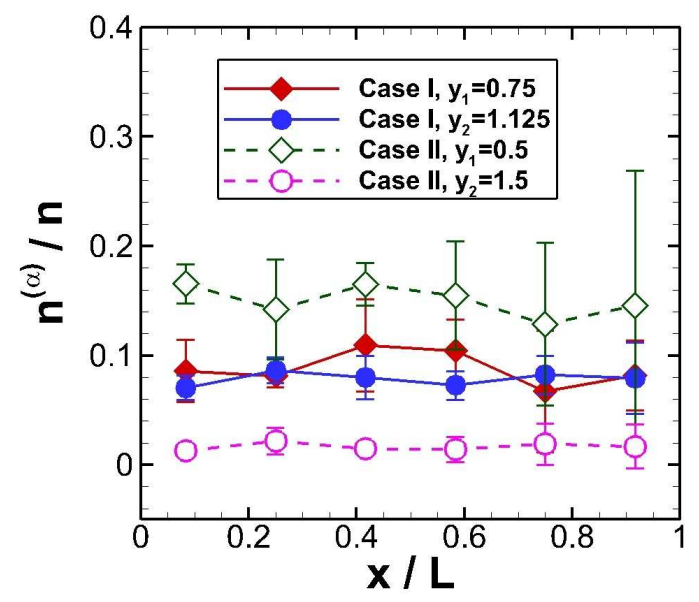

Figure 1: Number density profile of particle classes along the mean flow coordinate.

2015). Note that the grid resolution requirement to capture the boundary layer around the smallest particle in the configuration limits the range of particle size ratio in our simulations. The grid resolution used for these simulations are $\langle d\rangle=30 \Delta x$ which is appropriate for grid-independent results. In order to account for the statistical variability in particle arrangements, we also perform three independent realizations for each case. We start our simulations with fixed particle assemblies until flow structures are formed and the relative error in the mean drag force reduces to less that $10^{-6}$. Then we release the particles and let them evolve under the influence of hydrodynamic and collisional forces they experience, as described by Eqs. 10 and 11.

\subsection{Homogeneity of particle configuration}

In order to ensure that the particle configurations in our simulations remain statistically homogeneous, we compute the particle number density along the mean flow coordinate in the computational box, which is the aver- 
age number of particles per unit volume. To reduce statistical variability, the data have been averaged over six time intervals separated from each other by normalized time $t\langle d\rangle /|\langle\mathbf{W}\rangle|=5$, after the system reaches a steady state. The number density profiles in Fig. 1 indicate that both particle classes are uniformly distributed along the mean flow direction with no accumulation of particles. The radial distribution function (RDF) can also be used to identify preferential accumulation of neighbor particles. The RDF represents the probability of finding another particle at the vicinity of a test particle with separation $r$. RDF's from our simulations (not shown here) did not indicate any deviation from the initial condition. This again confirms that the particle configurations in our freely evolving simulations remain homogeneous for flow conditions considered here $\left(\phi=0.3, R e_{m}=50, \rho^{(p)} / \rho^{(f)}=1000\right.$, with elastic particles).

At higher solid-phase volume fractions, the caging effect, in which particles are trapped by their neighbors, is initiated by formation of microscopic or mesoscopic particle clusters in the suspension. These structures cause the particle distribution to deviate from homogeneity. Experimental studies of an air-driven bidisperse configuration (Abate and Durian, 2006) and also vertically vibrated monodisperse grains (Reis et al., 2007) indicate that the caging effect takes place near the solid-phase packing limit. In our cases, the configurations are much below the packing limit. Therefore, the caging phenomenon is not a matter of concern here, and particles are indeed homogeneously distributed. 


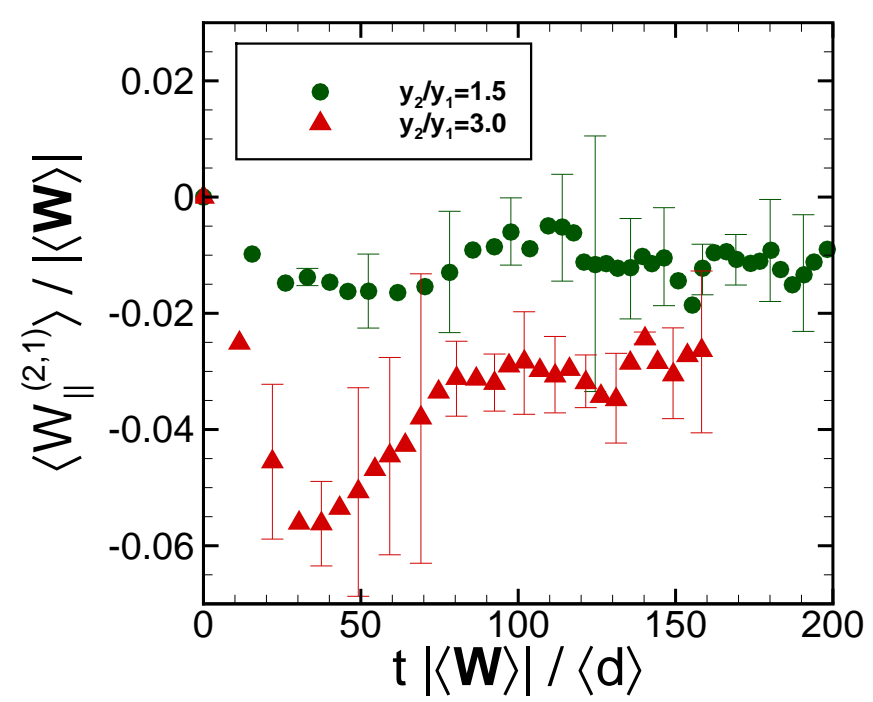

Figure 2: Prediction of slip velocity between two particle size classes parallel to the mean flow obtained from PR-DNS.

\subsection{Slip velocity between particle size classes}

We can directly track the evolution of the axial component of the slip velocity between the two particle size classes that is governed by Eq. 4, as shown by symbols in Fig. 2 for Case I and Case II where the size ratios are $y_{2} / y_{1}=1.5$ and 3.0 , respectively. As time progresses, the axial component of the slip velocity between the two size classes grows in magnitude due to the differences between the gas-particle drag and particle-particle drag forces on each particle size class. This mean slip velocity between the two particle size classes indicates that there is a particle mass flux even in a homogeneous suspension in the absence of gradients in the number density or granular temperature, or external forces (Garzó et al., 2007). This shows how segregation in gas-solid flow is different from granular flows. 


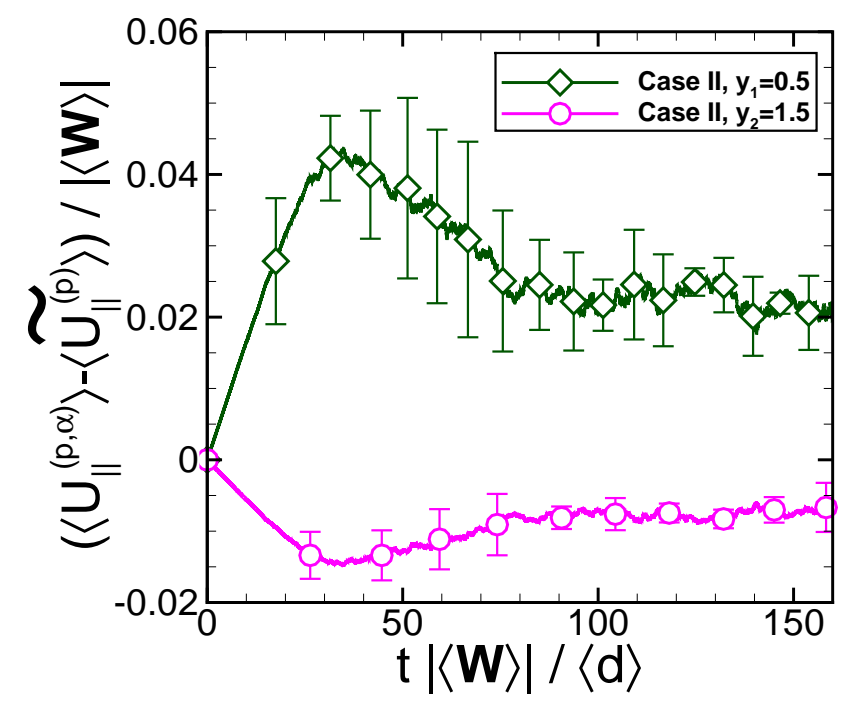

Figure 3: Evolution of normalized difference between axial component of particle size class mean velocities and mass-weighted particles mean velocity $\left\langle\widetilde{\mathbf{u}^{(p)}}\right\rangle$. Normalization is by the mean slip velocity $|\langle\mathbf{W}\rangle|=\left|\left\langle\widetilde{\mathbf{u}^{(p)}}\right\rangle-\left\langle\mathbf{u}^{(f)}\right\rangle\right|$.

The transient region in the evolution of the mean slip velocity between two particle size classes in Fig. 2 indicates that there exists a peak value in the mean slip velocity difference followed by a reduction before the steady value is attained. This behavior can be better understood by analyzing the evolution of the mean momentum for each particle size class described by Eq. 2 since it is the difference in the mean velocities of each size class $\left\langle\mathbf{u}^{(\alpha)}\right\rangle$ that results in $\left\langle\mathbf{W}^{(\beta, \alpha)}\right\rangle$. The rate of change of $\left\langle\mathbf{u}^{(\alpha)}\right\rangle$ is determined by the balance of terms on the right-hand side of Eq. 2 representing gas-particle drag and particleparticle drag forces. Since we start our freely evolving simulations after the hydrodynamic field characterized by a desired mean slip Reynolds number is developed, the mean gas-particle drag on each particle size class is already 
non-zero. Since the particle-particle drag force is negligible at the onset of freely moving suspension simulations that particles are initially at rest, the mean gas-particle drag force on each particle size class acts as a source of mean momentum on the right-hand side of Eq. 2. This source gives rise to the monotonic increase of the species diffusion velocity for each particle size class (difference between the mean velocity of particle size classes and the mass-weighted solid mixture velocity) as shown in Fig. 3 for Case II. This figure represents the evolution of the particle size class mean velocity with respect to the mass-weighted particle velocity $\left\langle\widetilde{\mathbf{u}^{(p)}}\right\rangle$. After the initial transition $(t|\langle\mathbf{W}\rangle| /\langle d\rangle>50)$ during which each particle size class attains a finite species diffusion velocity, collisions between particles belonging to different size classes become more frequent. As a result of momentum transfer from one particle size class to another due to particle collisions, the particleparticle drag force becomes comparable to the gas-particle drag force and acts as a sink term in Eq. 2. This sink of mean momentum hinders further increase of $\left\langle\mathbf{u}^{(\alpha)}\right\rangle$ (cf. Fig. 3), and once it balances the gas-particle drag force, the mean velocity of each particle size class reaches a steady value. The difference between the mean velocities of the two particle size classes then determines their corresponding mean relative motion.

It should be noted that although the mean slip velocity between particle classes is determined by a balance of acceleration due to pressure gradient, hydrodynamic force and particle-particle drag (see Eq. 4), this mean slip is small in both cases we considered in this study. One might be led to conclude that the particle-particle drag is significant (in order to balance the gas-particle drag term) even when the particle-particle slip velocity is 
small. To address this concern, for the two freely evolving cases considered in this study, we compare the average particle-particle drag force with the gas-particle drag in Table 2. The steady value of the gas-particle drag is reported at the end of the simulation, while the particle-particle drag is time-averaged in order to reduce the statistical variability. To calculate the particle-particle drag, the contact force is first averaged over all particles belonging to the same particle class, and then these averaged contact forces are time-averaged. In other words

$$
\begin{gathered}
\left\langle\mathbf{F}_{\text {coll }}^{(\beta \rightarrow \alpha)}\right\rangle=\frac{\sum_{i=1}^{N_{\alpha}} \mathbf{F}_{\text {coll }}^{(\beta \rightarrow \alpha)}}{N_{\alpha}}, \\
\left\langle\widetilde{\mathbf{F}_{\text {coll }}^{(\beta \rightarrow \alpha)}}\right\rangle=\frac{\sum_{j=1}^{N_{T}}\left\langle\mathbf{F}_{\text {coll }}^{(\beta \rightarrow \alpha)}\right\rangle \Delta t_{j}}{\sum_{j=1}^{N_{T}} \Delta t_{j}},
\end{gathered}
$$

where $N_{T}$ represents the number of time steps in the interval over which the time-averaging has been performed, and $\Delta t_{j}$ corresponds to the time interval of $j$ th time step. It should be noted that the above time-averaging accounts for approximately the last 40 normalized time units, where the reference time unit is $\langle d\rangle /|\langle\mathbf{W}\rangle|$. The above definition provides the time-averaged particleparticle drag force per particle acting on class $\alpha$ from class $\beta$. To be consistent with gas-particle drag, particle-particle drag forces are finally normalized by the Stokes drag expression evaluated at the mean slip Reynolds number, i.e. $\left.F_{S t}=3 \pi d_{\alpha} \mu^{(f)}(1-\phi)|\langle\mathbf{W}\rangle|\right)$.

The comparison of forces in Table 2 shows that the particle-particle drag on each particle class is much smaller than the corresponding gas-particle drag. However, it should be noted that the drag forces in Eq. 4 are per unit volume. Since the solid-phase densities of the two classes are the same the first term on the right-hand-side of Eq. 4 becomes zero. The second term 
Table 2: Comparison of steady gas-particle and particle-particle drag forces for the freely evolving cases. The second and third columns represent the gas-particle drag on each particle size class, the fourth and fifth columns represent the particle-particle drag force on each particle size class obtained from PR-DNS results, and the last two columns correspond to the particle-particle drag obtained from the model of Syamlal (1987) using the mean slip velocity between the two particle size classes from PR-DNS results for the two cases (cf. Fig. 2 of the MS). The gas-particle drag forces are reported at the end of the simulation corresponding to steady state (the values are subsequently averaged over five realizations), while the particle-particle drag forces are time-averaged over the last 40 normalized time unit to reduce statistical variability. All drag forces are given on per-particle basis normalized by the Stokes $\operatorname{drag} F_{S t}=3 \pi d_{i} \mu^{(f)}(1-\phi)|\langle\mathbf{W}\rangle|,(i=\alpha, \beta)$.

\begin{tabular}{lcccccc}
\hline Cases & $\left\langle F_{h}^{(\alpha)}\right\rangle$ & $\left\langle F_{h}^{(\beta)}\right\rangle$ & $\left\langle F_{\text {coll,DNS }}^{(\beta \rightarrow \alpha)}\right\rangle$ & $\left\langle F_{\text {coll, DNS }}^{(\alpha \rightarrow \beta)}\right\rangle$ & $\left\langle F_{\text {coll,S }}^{(\beta \rightarrow \alpha)}\right\rangle$ & $\left\langle F_{\text {coll,S }}^{(\alpha \rightarrow \beta)}\right\rangle$ \\
\hline Case I & 12.60 & 21.67 & -2.91 & 2.18 & -0.66 & 0.49 \\
Case II & 7.73 & 32.24 & -2.70 & 8.21 & -1.00 & 3.07 \\
\hline
\end{tabular}

on the right-hand side (RHS) is the difference between the mean interphase momentum transfers (gas-particle drag) divided by the corresponding bulk density (this term represents the difference between the mean accelerations of particle classes from the interphase momentum transfers). At steady state this balances the acceleration originating from the particle-particle drag in the third term on the RHS of Eq. 4. The RHS of this equation for Cases I and II are, respectively, computed from the PR-DNS data provided in Table 2, which are:

$$
\begin{aligned}
\operatorname{RHS}_{\text {Case I }} & =\frac{1}{1000}\left[\left(\frac{0.4685}{0.2243}-\frac{0.2262}{0.0747}\right)+0.0684\left(\frac{1}{0.2243}+\frac{1}{0.0747}\right)\right] \\
& =\frac{-0.9407+1.2206}{1000}
\end{aligned}
$$


and

$$
\begin{aligned}
\text { RHS }_{\text {Case II }} & =\frac{1}{1000}\left[\left(\frac{0.3438}{0.2209}-\frac{0.3333}{0.0748}\right)+0.1432\left(\frac{1}{0.2209}+\frac{1}{0.0748}\right)\right] \\
& =\frac{-2.8995+2.5627}{1000}
\end{aligned}
$$

where $\rho^{(p)}=1000$, and $\phi^{(\alpha)}=0.0747, \phi^{(\beta)}=0.2243$ for Case I, while $\phi^{(\alpha)}=0.0748, \phi^{(\beta)}=0.2209$ for Case II. Again we see that the particleparticle drag in this balance (0.0684 for Case I and 0.1432 for Case II) is smaller than the corresponding gas-particle drag values. We conclude that it is the fact that the gas-particle drag contributions (scaled by their corresponding volume fractions) subtract from each other, while particle-particle drag contributions (also scaled by their corresponding class volume fractions) add to each other that results in a low magnitude particle-particle drag balancing the higher magnitude gas-particle drag in the mean particle class slip velocity equation at steady state. At the end of this section we find that accurate modeling of both the fluid-particle drag and particle-particle drag is necessary for accurate prediction of the particle mass flux that leads to segregation.

\section{Assessment of bidisperse gas-particle drag models in predicting mean slip velocity between two particle size classes}

We examine the capabilities of existing two-fluid closure models to capture the particle mass flux and mean slip velocity between particle size classes. The focus of the current study is mainly on the influence of the hydrodynamic force. Therefore, we use the gas-particle drag models proposed by Syamlal and O'Brien (1987), which is widely used in CFD packages such as MFIX, as 
Table 3: Bidisperse gas-particle drag models used in TFM CFD simulations. These drag are given for either the mean interphase transfer $\mathbf{S}_{\mathrm{h}}^{(\alpha)}$ or the total gas-particle drag force that also includes the contribution from the mean pressure gradient $\mathbf{F}^{(\alpha)}=$ $\mathbf{S}_{\mathrm{h}}^{(\alpha)}-\langle\boldsymbol{\nabla} p\rangle V^{(\alpha)}$. These drag models represent the mean drag force per particle normalized by the Stokes drag force, that is $F_{S t}=3 \pi d_{\alpha} \mu^{(f)}(1-\phi)|\langle\mathbf{W}\rangle|$.

\begin{tabular}{|c|c|c|}
\hline Model & Source & Formulation \\
\hline $\mathrm{SO}$ & Syamlal and O'Brien (1987) & $\left\langle S_{\mathrm{h}}^{(\alpha)}\right\rangle=y_{\alpha} \frac{R e_{m}}{24} \frac{C_{D 0}^{(\alpha)}}{\left(1-\phi^{(p)}\right) V_{\alpha}^{2}}\left(\frac{\left|\left\langle\mathbf{W}^{(\alpha)}\right\rangle\right|}{|\langle\mathbf{W}\rangle|}\right)^{2}$, \\
\hline BVK & Beetstra et al. (2007) & $\begin{array}{l}\left\langle F_{\mathrm{h}}^{(\alpha)}\right\rangle=\left\langle F_{\mathrm{h}}^{(\text {mono })}\right\rangle\left(y_{\alpha}\left(1-\phi^{(p)}\right)+y_{\alpha}^{2} \phi^{(p)}\right) \\
\left\langle F_{\mathrm{h}}^{(\text {mono })}\right\rangle=\frac{10 \phi^{(p)}}{\left(1-\phi^{(p)}\right)^{3}}+\left(1-\phi^{(p)}\right)\left(1+1.5 \sqrt{\phi^{(p)}}\right) \\
+\frac{0.413 \operatorname{Re}_{m}}{24\left(1-\phi^{(p)}\right)^{3}}\left(\frac{\left(1-\phi^{(p)}\right)^{-1}+3 \phi^{(p)}\left(1-\phi^{(p)}\right)+8.4 \operatorname{Re}_{m}^{-0.343}}{1+100^{3 \phi}(p)}\right) \\
\operatorname{Re}_{m}^{-\left(1+4 \phi^{(p)}\right) / 2}\end{array}$ \\
\hline RDY & Rong et al. (2014) & $\begin{array}{l}\left\langle S_{\mathrm{h}}^{(\alpha)}\right\rangle=y_{\alpha}\left\langle S_{\mathrm{h}}^{(\text {mono })}\right\rangle\left(\frac{\left|\left\langle\mathbf{W}^{(\alpha)}\right\rangle\right|}{\mid\langle\mathbf{W}\rangle}\right)^{2} \\
\left\langle S_{\mathrm{h}}^{(\text {mono })}\right\rangle=\frac{R e_{m}}{24} C_{D 0}\left(1-\phi^{(p)}\right)^{-\beta} \\
\beta=2.65\left[\left(1-\phi^{(p)}\right)+1\right]-\left[5.3-3.5\left(1-\phi^{(p)}\right)\right] \\
\times\left(1-\phi^{(p)}\right)^{2} \exp \left[-0.5\left(1.5-\log \left(R e_{m}\right)\right)^{2}\right]\end{array}$ \\
\hline MTS & Present study & $\begin{array}{l}\left\langle F_{\mathrm{h}}^{(\alpha)}\right\rangle=\left\langle F_{\mathrm{h}}^{(\text {mono) }}\right\rangle\left(y_{\alpha}\left(1-\phi^{(p)}\right)+y_{\alpha}^{2} \phi^{(p)}\right) \\
\left\langle F_{\mathrm{h}}^{(\mathrm{mono})}\right\rangle=\frac{F_{\mathrm{isol}}}{\left(1-\phi^{(p)}\right)^{3}}+F_{\phi^{(p)}}+F_{\phi^{(p)}, R e_{m}} \\
F_{\text {isol }}=1+0.15 \operatorname{Re}_{m}^{0.867} \\
F_{\phi^{(p)}}=\frac{5.81 \phi^{(p)}}{\left(1-\phi^{(p)}\right)^{3}}+0.48 \frac{\phi^{(p) 1 / 3}}{\left(1-\phi^{(p)}\right)^{4}} \\
F_{\phi^{(p)}, \operatorname{Re}_{m}}=\phi^{(p) 3} \operatorname{Re}_{m}\left(0.95+\frac{0.61 \phi^{(p) 3}}{\left(1-\phi^{(p)}\right)^{2}}\right)\end{array}$ \\
\hline
\end{tabular}

well the drag models proposed by Kuipers' group (van der Hoef et al., 2005; Beetstra et al., 2007; van der Hoef, 2007) and Rong et al. (2014) in the gas and solid-phase mean momentum equations 1 and 2. To close these set of equations, a particle-particle drag model is also needed to account for the mean momentum transfer between two particle size classes $\left\langle\mathbf{f}_{\text {coll }}^{(\beta \rightarrow \alpha)}\right\rangle$ due to particle collisions. Since the focus of the current study is on the contribution from the gas-particle drag to the mean slip velocity between two particle size classes, we use only the particle-particle drag model of Syamlal (1987), that 
has the following form:

$$
\left\langle\mathbf{f}_{\text {coll }}^{(\beta \rightarrow \alpha)}\right\rangle=\frac{3}{4}\left(1+\varepsilon_{\alpha \beta}\right) \frac{\phi^{(\alpha)} \rho^{(\alpha)} \phi^{(\beta)} \rho^{(\beta)}}{\rho^{(\alpha)} d_{\alpha}^{3}+\rho^{(\beta)} d_{\beta}^{3}}\left(d_{\alpha}+d_{\beta}\right)^{2} g_{0}\left|\left\langle\mathbf{W}^{(\beta, \alpha)}\right\rangle\right|\left\langle\mathbf{W}^{(\beta, \alpha)}\right\rangle,
$$

where $\varepsilon_{\alpha \beta}$ is the coefficient of restitution in a collision between a colliding pair of particles belonging to classes $\alpha$ and $\beta$, and $g_{0}$ is the value of radial distribution function at contact represented by the model of Lebowitz (1964). It is worth mentioning that this gas-particle drag model has no dependence on the velocity distribution of each particle size class. This model assumes that the velocity distributions are delta-functions that correspond to nonzero mean velocities with zero variance. Other models like the one proposed by Louge et al. (1991) do not generalize to this case. However, for this study we do not change the particle-particle drag model and investigate the effect of gas-particle drag model.

For a better insight into the aforementioned gas-particle drag models, they are presented in Table 3. For the sake of convenience, we refer to these models, respectively, by SO, BVK, and RDY from hereon. These gasparticle drag models are presented based on the average drag force per particle normalized by the Stokes drag force on a particle in size class $\alpha$, i.e. $F_{S t}=3 \pi d_{\alpha} \mu^{(f)}(1-\phi)|\langle\mathbf{W}\rangle|$. It should be noted that the SO model is originally given based on the volumetric mean drag force. However, for ease of comparison with other drag models in Table 3, it is shown here on a per particle basis (cf. Eq. 16). Similarly, the gas-particle drag model of RDY for TFM simulation of freely evolving suspensions translates to the form presented in Table 3 .

It is evident that the $\mathrm{SO}$ model is based on purely inertial scaling, i.e. $F \sim$ 
$\rho^{(f)}|\langle\mathbf{W}\rangle|^{2} / 2$. However, the BVK model relates the particle drag on each size class to that of an equivalent monodisperse drag model by a quadratic expression in $y_{\alpha}$. The equivalent monodisperse drag in BVK model is a complex function of $\phi^{(p)}$ and $R e_{m}$ obtained from Lattice-Boltzmann PR-DNS (Beetstra et al., 2007). The RDY drag model is similar to that of SO in its inertial scaling. Nevertheless, a different model is used for the monodisperse drag law (Rong et al., 2013).

It should be noted that the gas-particle drag models are presented either for the interphase momentum transfer $\left\langle\mathbf{s}_{\mathrm{h}}^{(\alpha)}\right\rangle$ or the total hydrodynamic drag force $\left\langle\mathbf{f}_{\mathrm{h}}^{(\alpha)}\right\rangle$ which also includes the contribution from the mean pressure gradient. Furthermore, these drag models are given either based on mean per unit volume basis or mean per particle basis. In the current study, the former are denoted with small letters $\left(\left\langle\mathbf{s}_{\mathrm{h}}^{(\alpha)}\right\rangle\right.$ and $\left.\left\langle\mathbf{f}_{\mathrm{h}}^{(\alpha)}\right\rangle\right)$, while the latter are referred to by capital letters $\left(\left\langle\mathbf{S}_{\mathrm{h}}^{(\alpha)}\right\rangle\right.$ and $\left.\left\langle\mathbf{F}_{\mathrm{h}}^{(\alpha)}\right\rangle\right)$. These quantities are related to each other through the following expressions:

$$
\begin{aligned}
& \left\{\begin{array}{l}
\left\langle\mathbf{F}_{\mathrm{h}}^{(\alpha)}\right\rangle=-\langle\mathbf{g}\rangle V^{(\alpha)}+\left\langle\mathbf{S}_{\mathrm{h}}^{(\alpha)}\right\rangle, \\
\left\langle\mathbf{f}_{\mathrm{h}}^{(\alpha)}\right\rangle=-\langle\mathbf{g}\rangle \phi^{(\alpha)}+\left\langle\mathbf{s}_{\mathrm{h}}^{(\alpha)}\right\rangle,
\end{array}\right. \\
& \left\{\begin{array}{l}
\left\langle\mathbf{f}_{\mathrm{h}}^{(\alpha)}\right\rangle=n^{(\alpha)}\left\langle\mathbf{F}_{\mathrm{h}}^{(\alpha)}\right\rangle \\
\left\langle\mathbf{s}_{\mathrm{h}}^{(\alpha)}\right\rangle=n^{(\alpha)}\left\langle\mathbf{S}_{\mathrm{h}}^{(\alpha)}\right\rangle
\end{array}\right.
\end{aligned}
$$

where $V^{(\alpha)}$ is the volume of a particle in size class $\alpha$, and $n^{(\alpha)}$ is $\alpha^{\text {th }}$ size class particle number density.

We solve the mean momentum equations 1 and 2 with the use of gasparticle drag models in Table 3 and the particle-particle drag model in Eq. 14 for the cases I and II presented in Section 2. The driving force in the mean 


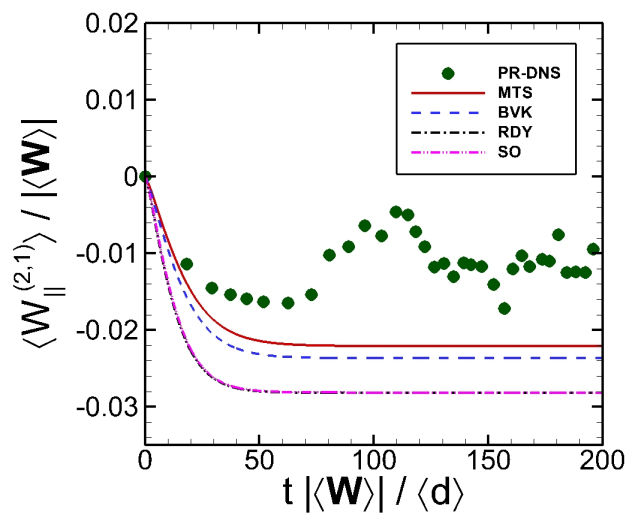

(a) Case I

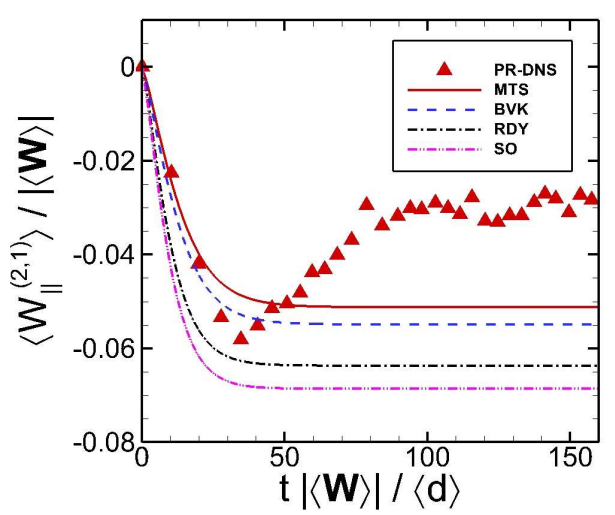

(b) Case II

Figure 4: Comparison of the mean slip velocity between two particle size classes obtained from PR-DNS with those obtained from solving the mean momentum equations (cf. Eqs. 1 and 2) closed with different gas-particle drag models (see legends) and the particle-particle drag model given by Eq. 14 .

momentum equations is the mean pressure gradient $\langle\mathrm{g}\rangle$. We set the mean pressure gradient such that the mean slip Reynolds number at the steady state matches that of the PR-DNS. The predicted mean slip velocity between the two particle size classes are then compared with our PR-DNS results. It is evident from Fig. 4 that the steady values significantly deviate from 100\% for BVK model to $200 \%$ for RDY and SO models compared to PR-DNS data. In addition, the evolution of the mean slip velocity obtained from the closure models is not able to correctly capture the transient behavior when compared to the PR-DNS results. The difference in the steady value of the mean slip velocity between two size classes, and also the inability of correctly predicting the transient evolution may indicate that either the gas-particle drag or the particle-particle drag model is not accurately predictive in this particular gas- 
solid flow problem. To validate this assumption, in the following section we analyze the gas-particle drag force in bidisperse suspensions in more detail. The analysis of particle-particle drag force, however, is not covered in the present study.

\section{Gas-particle drag in a bidisperse gas-solid suspension}

We use fixed bidisperse particle assemblies in PR-DNS of homogeneous gas-solid flows to extract the average mean drag force on each particle size class. Kuipers' group (van der Hoef et al., 2005; Beetstra et al., 2007; Sarkar et al., 2009) and Rong et al. (2014) also used fixed particle assemblies to propose their gas-particle drag models for monodisperse and bidisperse suspensions. Xu and Subramaniam (2010), Tenneti et al. (2011) and Mehrabadi et al. (2015) argued that the use of fixed particle assemblies is legitimate in suspensions containing massive particles with high particle Stokes number because the time it takes for changes in the particle spatial configuration is much greater that the momentum relaxation time. Tenneti et al. (2011) and Mehrabadi et al. (2015), respectively, showed that drag and the level of gasphase velocity fluctuations are similar between fixed particle assemblies and frely evolving suspensions. Fixed particle assemblies are less computationally expensive compared to simulations of freely evolving suspensions. In addition, the dimensionality of the parameter space is now reduced because the density ratio is no longer a parameter. We perform PR-DNS of these particle assemblies until a steady state is obtained. Then the average drag force per particle for each size class is computed and normalized by the Stokes drag force. 


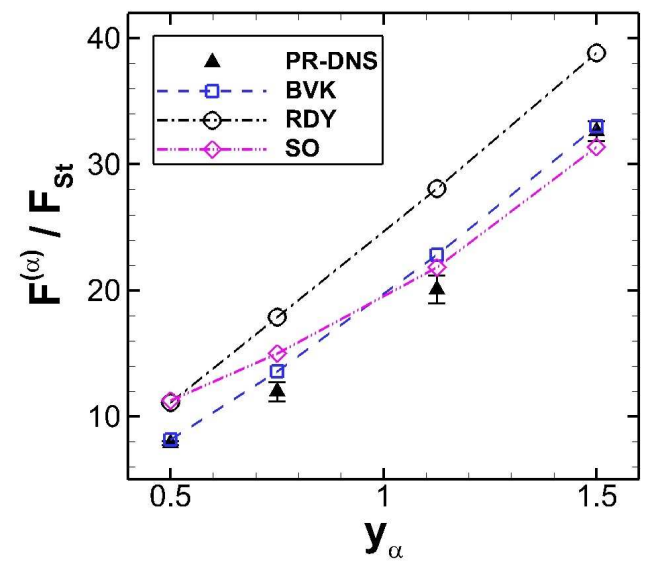

(a)

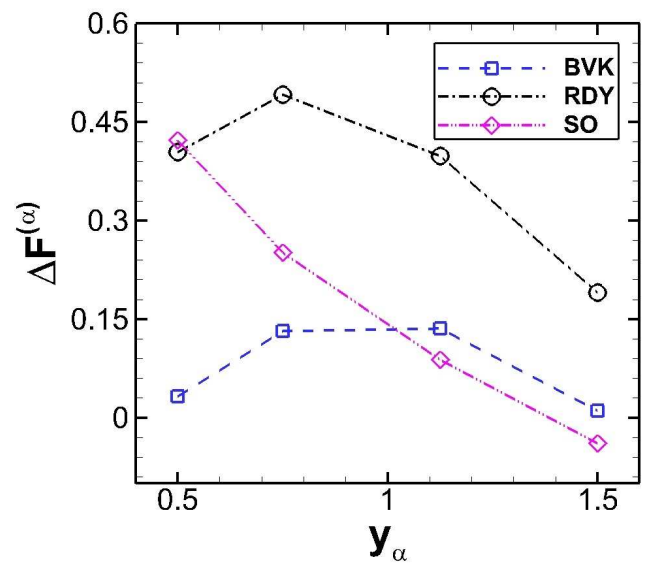

(b)

Figure 5: (a) Comparison of the gas-particle drag force on each particle class predicted by closure models (SO, BVK, and RDY) for flow parameters associated with Cases I and II (cf. 1) with those obtained from PR-DNS. (b) The relative error in gas-particle drag models with respect to PR-DNS data.

Our PR-DNS data set includes simulations with $R e_{m}$ values of 50,65 , 75 , and 100 at total volume fractions $0.1,0.2,0.3$, and 0.4 . At each volume fraction, the volume fraction ratio $\left(\phi^{(2)} / \phi^{(1)}\right)$ is varied from 1 to 6 and the diameter ratio $\left(d^{(2)} / d^{(1)}\right)$ from 1.5 to 4 . In order to account for the statistical variability in particle configurations, four different realizations are considered for each case. It should be noted that although our simulation data set covers a range of solid-phase volume fractions and mean slip Reynolds numbers, due to the similarity of results we only present those for $R e_{m}=50$ and 75 , and $\phi^{(p)}=0.3$ and 0.4 .

We first compare the gas-particle drag force obtained from our PR-DNS of fixed particle assemblies corresponding to particle size classes used in freely evolving suspensions of cases I and II with the predictions obtained from SO, 
BVK and RDY at the same flow parameter in Fig. 5. Note that the $y_{\alpha}$ values used in the freely evolving suspensions are 0.5 and 1.5 for Case I, and 0.75 and 1.125 for Case II at $\phi^{(p)}=0.3$ and $R e_{m}=50$.

The comparison in Fig. 5 reveals that the SO drag model significantly over-predicts the drag force on smaller particle size classes with the maximum difference being about 45\%, whereas the prediction improves with increasing $y_{\alpha}$. Over-prediction of the gas-particle drag force on smaller particles gives rise to higher mean acceleration that leads to higher mean velocity for the smaller size class. This in turn appears as higher slip velocity between the two particle size classes observed in Fig. 4. The RDY drag model over-predicts the drag force over the entire range of $y_{\alpha}$ with the difference ranging from $20 \%$ to $50 \%$ in Fig.5. These over-predictions of the drag force, consequently, do give rise to inaccurate slip velocity between two particle size classes in Fig. 4. The BVK model provides closer drag prediction compared with the PR-DNS data with the maximum difference being about 15\% in Fig. 5. Therefore, it provides a better mean slip velocity prediction between the two particle size classes in Fig. 4 as compared to the SO and RDY drag models.

Inaccuracy of existing gas-particle drag models in predicting the mean slip velocity difference motivates us to propose an improved gas-particle drag model based on the PR-DNS data of fixes particle assemblies. We use the normalized gas-particle drag correlation of BVK for bidisperse suspension which has the following form:

$$
\frac{\left\langle F_{\mathrm{h}}^{(\alpha)}\right\rangle}{\left\langle F_{\mathrm{h}}^{(\text {mono })}\right\rangle}=y_{\alpha}\left(1-\phi^{(p)}\right)+y_{\alpha}^{2} \phi^{(p)},
$$

where $\left\langle F_{\mathrm{h}}^{(\mathrm{mono})}\right\rangle$ is the equivalent monodisperse drag model at the same 


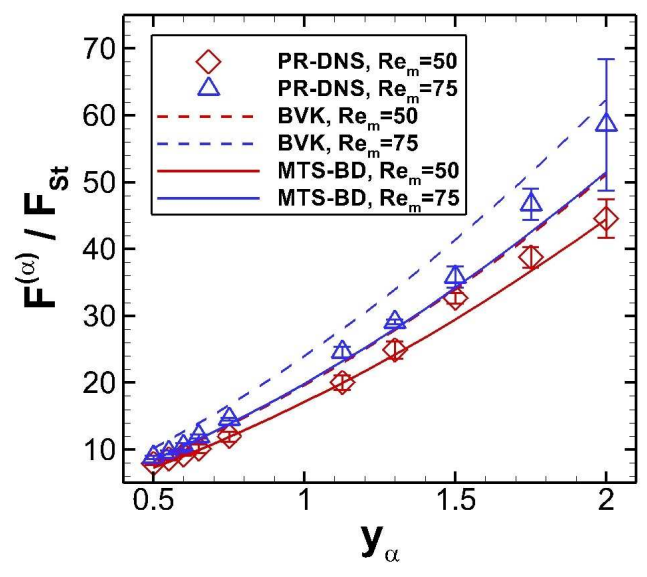

(a) $\phi^{(p)}=0.3$

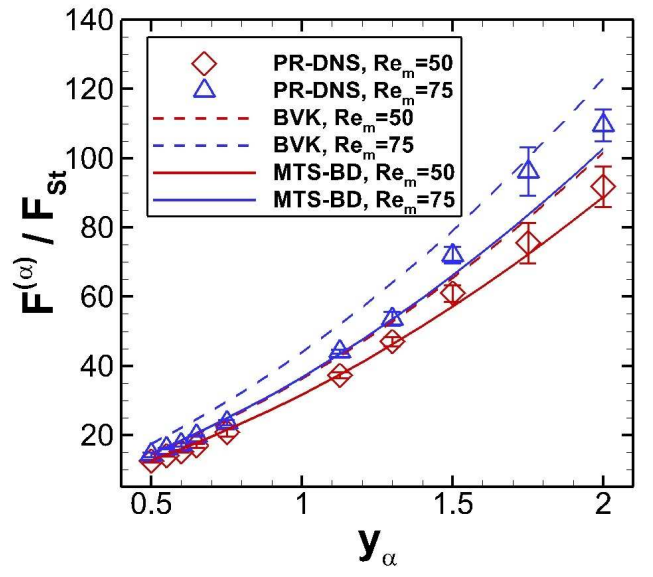

(b) $\phi^{(p)}=0.4$

Figure 6: Plot showing the normalized force obtained from PR-DNS of flow past fixed bidisperse particle assemblies (symbols) at a total volume fraction of (a) $\phi^{(p)}=0.3$ and (b) $\phi^{(p)}=0.4$ for two mean flow Reynolds numbers $\left(R e_{m}=50\right.$ and 75$)$. The normalized force is reported by averaging over 4 realizations and the error bars show $95 \%$ confidence intervals in the estimation of the normalized force. PR-DNS data are compared with the MTS gas-particle drag model (solid lines) as well as the BVK drag model (dashed lines).

total solid-phase volume fraction and mean slip Reynolds number. This expression indicates that the bidisperse drag and the equivalent monodisperse drag forces can be related to each other through a simple function of total solid-phase volume fraction and size class diameter to Sauter mean diameter ratio. van der Hoef et al. (2005) proposed the above correlation based on the condition that in Stokes flow, the total volumetric drag force in a bidisperse suspension should be equal to the volumetric drag force of a monodisperse system with particle diameter as $\langle d\rangle$. The above correlation has also been tested for intermediate and high Reynolds number suspensions.

The above correlation accompanied by the monodisperse drag model of 
Beetstra et al. (2007) provides the basis for the BVK bidisperse drag model given in Table 3. As observed in Fig. 6, the BVK model (represented by dashed lines) is not able to quantitatively predict our PR-DNS data. The maximum difference between the two data sets is more than $15 \%$ for particle size classes $y_{\alpha}<\langle d\rangle$. The monodisperse drag model of Beetstra et al. (2007) relies on drag force obtained from a grid resolution of $d / \Delta x=17.5$ for $\phi^{(p)} \leq 0.3$, and grid resolutions $d / \Delta x=17.5$ and 25 for $\phi^{(p)}>0.3$, to simulate Reynolds numbers ranging from 21 to 1000. However, other studies indicate that the grid resolution requirement increases with both mean slip Reynolds number and solid volume fraction (Tenneti et al., 2011). As the mean slip Reynolds number increases, the thickness of particle boundary layers decrease $\left(\delta \sim 1 / \sqrt{R e_{m}}\right)$ and higher resolution is needed to resolve these adequately and to accurately compute the drag force. As the solid volume fraction increases, the distance between particle surfaces decreases and again higher resolution is needed to resolve the flow in the interstices adequately.

Here, we use Eq. 17 jointly with the monodisperse drag model of Tenneti et al. (2011), which has the following form:

$$
\left\langle F_{\mathrm{h}}^{(\mathrm{mono})}\right\rangle=\frac{F_{\text {isol }}\left(R e_{m}\right)}{(1-\phi)^{3}}+F_{\phi}(\phi)+F_{\phi, R e_{m}}\left(\phi, R e_{m}\right)
$$

where, $F_{\text {isol }}$ is the drag force acting on an isolated sphere moving in an unbounded medium. The drag on an isolated sphere is taken to be the correlation proposed by Schiller and Naumann (1935). The remaining two terms 
in Eq. 18 are given by

$$
\begin{aligned}
F_{\phi}(\phi) & =\frac{5.81 \phi}{(1-\phi)^{3}}+0.48 \frac{\phi^{1 / 3}}{(1-\phi)^{4}} \\
F_{\phi, R e_{m}}\left(\phi, R e_{m}\right) & =\phi^{3} \operatorname{Re}_{m}\left(0.95+\frac{0.61 \phi^{3}}{(1-\phi)^{2}}\right) .
\end{aligned}
$$

If we use Eqs. 17 and 18, the prediction of gas-particle drag force on each particle size class over the entire range of $y_{\alpha}$ improves. This improvement in predicting bidisperse gas-particle drag model in Fig. 6 arises from the fact that the monodisperse drag model of Tenneti et al. (2011) is based on highly resolved PR-DNS of gas-solid flow over the range of solid-phase volume fraction $0.1 \leq \phi^{(p)} \leq 0.5$ and the mean slip Reynolds number $0.01 \leq R e_{m} \leq 300$. Therefore, we recommend using Eq. 17 jointly with the monodisperse drag model of Tenneti et al. (2011) for bidisperse gas-particle drag in CFD calculations. We refer to this model as the MTS bidisperse gas-particle model.

\subsection{Comparison of drag force from closure models with PR-DNS of freely evolving suspensions}

It is of interest to compare the evolution of the gas-particle drag force obtained from PR-DNS of freely evolving suspension for Cases I and II in Section 4 with predictions of the closure models in Table 3. It should be noted that the BVK and MTS drag models assume that both size classes move with the same mean velocity, implying that the mean slip velocity between particle size classes is neglected. This is due to the fact that BVK and MTS models do not incorporate the slip velocity between particle size classes. However, since the steady size class diffusion velocity is about $2 \%$ of the mean slip velocity $|\langle\mathbf{W}\rangle|$ in these cases (cf. Fig. 3), the influence on the 
drag force is negligible according to Eqs. 17 and 18. It is worth mentioning that Holloway et al. (2010) proposed a bidisperse drag model by decomposing the gas-particle drag into a hydrodynamic part arising from the fluid-particle mean slip velocity, and fluid-mediated particle-particle part arising from the mean slip velocity between the two particle classes. Our analysis (not shown here) indicates that based on the values obtained from our PR-DNS for case I and II, the contribution from the fluid-mediated particle-particle drag is about $3 \%$ of the hydrodynamic part. Therefore, this model evaluation also indicates that the hydrodynamic drag due the particle-particle slip for the cases considered in our study cannot play a significant role in the gas-particle drag, and eventually in the segregation dynamics. Nevertheless, this may not always be true for cases where particle size classes have different material densities.

The comparison of the drag force from freely evolving PR-DNS with the drag models in Fig. 7 reveals that the difference in the drag force on smaller particle size classes $\left(y_{\alpha}=0.5\right.$ and 0.75$)$ predicted by MTS and BVK compared to PR-DNS results is about $5 \%$ to $8 \%$, while the difference ranges from $15 \%$ to $40 \%$ for SO and RDY models. However, BVK and SO models predict the drag for larger particles $\left(y_{\alpha}=1.125\right.$ and 3.0) at steady state with less than 5\% difference when compared with PR-DNS data, while this difference is about $8 \%$ for the MTS model and about $20 \%$ for the RDY model. This comparison indicates that the MTS and BVK drag models are more reliable for computing the gas-particle drag force in a freely evolving bidisperse suspension for the range of particle size classes considered in the current study (for size class density ratio of unity). However, it should be noted that none 
of these models incorporate any dependence of the steady drag on the fluctuating velocity of moving particles, such as recently proposed by Tang et al. (2016). Comparison with PR-DNS would be possible as such so-called dynamic drag laws from PR-DNS of freely evolving monodisperse suspensions are developed.

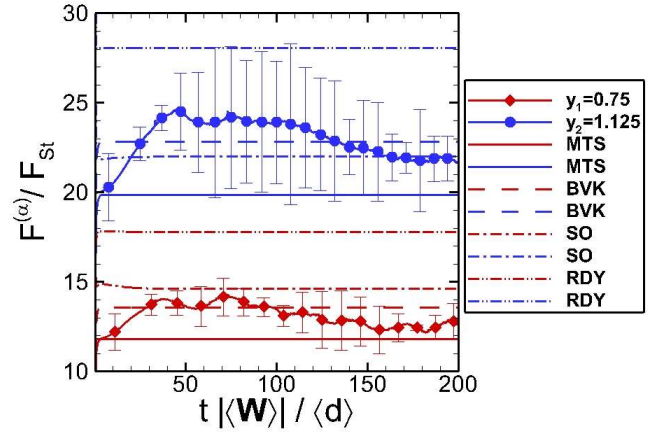

(a) Case I

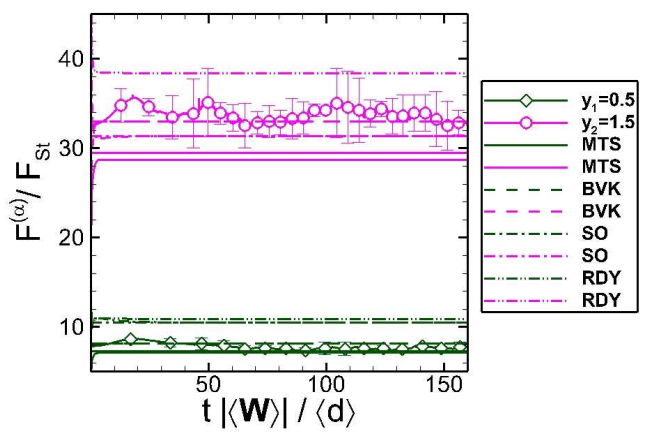

(b) Case II

Figure 7: Comparison of the mean gas-particle drag force per particle in each particle size class obtained from PR-DNS of freely evolving suspensions and those obtained from drag models described in Table 3. The symbols show the evolution of the drag with respect to normalized time, while the solid lines represent the drag for fixed assemblies. Note that line are colored with respect to the corresponding symbols. (a) Case I with size class diameters $y_{\alpha}=0.75$ and 1.125, (b) Case II with size class diameters $y_{\alpha}=0.5$ and 1.5.

We now examine the ability of the MTS drag model to predict the mean slip velocity between two particle classes, similar to the assessment we performed in Section 5 for other drag models. Therefore, we solve the mean momentum equations 1 and 2 with the MTS gas-particle drag model jointly with the particle-particle drag model of Syamlal (1987) for Cases I and II, and then compute the parallel component of $\left\langle\mathbf{W}^{(2,1)}\right\rangle$ along the mean flow direction. The comparison of the result with PR-DNS data as in Fig, 4 indi- 
cates that the MTS drag model has slightly improved the prediction of the mean slip velocity between two particle size classes (about 10\% improvement compared to BVK drag model).

\section{Discussion}

The development of drag models for bidisperse and polydisperse systems poses certain unique challenges that are not encountered in monodisperse systems. For a bidisperse suspension the drag force on each particle size class in general depends on the size ratio, density ratio, and also the mean particle class slip velocity with respect to the mean fluid velocity.

In the previous section, we used fixed bidisperse particle configurations to quantify the drag force on each particle size class. We then used the BVK bidisperse expression to relate the drag force on each particle class to the equivalent monodisperse suspension having the same total solid-phase volume fraction and mean slip Reynolds number based on Sauter mean diameter. This relation is solely a function of the particle size ratio $y_{\alpha}$ and solid-phase volume fraction $\phi^{(p)}$ that includes only geometrical information. No information about the size class inertia or dynamics of the bidisperse mixture is considered. In reality, particles are free to move and the difference between the mean velocities between particle size classes gives rise to the mean slip velocity between two particle size classes. However, Eq. 17 does not reflect any dependence the drag force may have on the mean slip velocity of each particle size class. Fixed particle assembly simulations have been used to study the effect of different mean slip velocity between particle size

classes (Yin and Sundaresan, 2009). However, Tenneti et al. (2011) showed 
that such simulations are not Galilean invariant, and could lead to significant error if performed at high mean slip Reynolds numbers.

It should be noted that for a given particle configuration and flow parameters $\left(\phi^{(p)}, \phi^{(2)} / \phi^{(1)}, d_{2} / d_{1}, \rho^{(2)} / \rho^{(1)}, R e_{m}\right)$, a specified particle size class mean slip velocity develops that is unknown a priori. Therefore, for a particular bidisperse gas-solid suspension there is no easy way to setup a suite of numerical simulations for populating the accessed values of $\left|\left\langle\mathbf{W}^{(\alpha)}\right\rangle\right| /|\langle\mathbf{W}\rangle|$ in the parameter space.

If the mean slip velocity between two particle size classes is negligible, then it is expected that the MTS or BVK drag models predict the gasparticle drag force of each particle size accurately. However, if in problems with notable difference in material densities of the particle size classes, the mean slip velocity between two particle size classes would be significant. Under these circumstances, the larger or heavier particles may experience an inertial flow regime, while the smaller or lighter particles may be in the Stokes flow regime. For such systems, the MTS or BVK drag models may not be able to correctly predict the drag force on each particle size class since they are solely based on $y_{\alpha}$ and $\phi^{(p)}$ relative to the equivalent monodisperse system. This is the condition where these models may break down. The SO drag model, on the other hand, includes the effect of the particle size class slip velocity through the term $\left(\left|\left\langle\mathbf{W}^{(\alpha)}\right\rangle\right| /|\langle\mathbf{W}\rangle|\right)^{2}$. Although there are differences in these two approaches in expressing the drag force on each particle size class, the appropriate form of a bidisperse drag model that can represent both geometrical and dynamical characteristics of a bidisperse suspension is still an open question. 
It may be inferred from the SO and either MTS or BVK drag models that a tentative form of a bidisperse drag model that includes both geometrical and inertial dynamics effects could have the form

$$
\left\langle F_{\mathrm{h}}^{(\alpha)}\right\rangle=f\left(y_{\alpha}, \frac{\left|\left\langle\mathbf{W}^{(\alpha)}\right\rangle\right|}{|\langle\mathbf{W}\rangle|}\right)\left\langle F_{\mathrm{h}}^{(\mathrm{mono})}\right\rangle .
$$

As a starting point, one path could be to take the SO drag model and modify the $C_{D 0}$ coefficient for a better match with the PR-DNS data from freely moving bidisperse gas-solid flow. The other path could be to start with MTS/BVK drag model and modify those by analogy to the SO drag model with the term $\left(\left|\left\langle\mathbf{W}^{(\alpha)}\right\rangle\right| /|\langle\mathbf{W}\rangle|\right)^{2}$. However, currently we did not follow these paths because they do not seem to be a completely rational approach that can be expected to result in a physics-based bidisperse drag model that covers a wide range of parameters.

The other route to improving the prediction of the mean slip velocity between the two particle size classes is to investigate particle-particle drag models and propose more accurate particle-particle drag models if needed. For instance, the model of Syamlal (1987) which is used for the analysis in this study (cf. Eq. 14) predicts a quadratic dependence. The particle-particle drag predicted by this model based on the flow parameters of case I and case II are presented in the Table 2. This comparison shows a significant difference between the predicted values by the model and those obtained from PRDNS. Although a more detailed analysis is required to address this difference, we believe that the particle velocity distribution characterized by non-zero particle granular temperature, plays a key role in the level of particle-particle drag (Louge et al., 1991). Note that the particle-particle drag model of 
Syamlal (1987) does not account for the particle velocity variance because it assumes a delta-function velocity distribution which is only dependent on the particle-particle mean slip velocity. Therefore, the difference between the PR-DNS results and the model predictions may be due to the fact that the granular temperature dependence of the gas-particle drag (as noted in the previous section) and particle-particle drag, although inherent in PR-DNS, is not accounted for by the models given in this study.

\section{Conclusions}

The slip velocity between two particle classes in a bidisperse gas-solid flow is the key signature of particle mass flux that in turn leads to segregation phenomena observed in fluidized beds. In this work, we used the PR-DNS methodology to quantify the slip velocity between particle classes in a homogeneous bidisperse gas-solid suspension at finite mean slip Reynolds number. Our simulations indicate that even in the absence of a body force, or gradients in particle number density or granular temperature, there exists a non-zero slip velocity between the two particle classes in a bidisperse suspension. This slip velocity arises from the difference in gas-particle drag and particle-particle drag forces on each particle class. We compared different bidisperse gas-particle drag models that are in use. While all of them reproduce the general trends, there are quantitative differences with PR-DNS data. In order to obtain a more accurate gas-particle drag model, we used fixed particle assemblies to perform PR-DNS of homogeneous bidisperse gassolid flow over a range of total solid-phase volume fractions $(0.1 \leq \phi \leq 0.4)$ and mean slip Reynolds number $\left(50 \leq R e_{m} \leq 100\right)$ with different particle 
size ratio $\left(0.5 \leq y_{\alpha} \leq 2.0\right)$ and volume fraction ratio $\left(1 \leq \phi^{(2)} / \phi^{(1)} \leq 6\right)$. Measurement of the mean drag force for each particle size class from our fixed PR-DNS results validates the correlation of Beetstra et al. (2007) in Eq. 17 for polydisperse gas-solid flow when used in conjunction with the equivalent monodisperse drag model of Tenneti et al. (2011). Comparison of the gasparticle drag force predicted from the MTS model with those obtained from PR-DNS of freely moving particles shows a good agreement for the range of particle size ratios provided in the current study. Using the MTS gas-particle drag model to solve the mean momentum equations of a bidisperse suspension also improves the prediction of the slip velocity between the two particle classes when compared to the predictions obtained using other gas-particle drag models. The unique challenges in developing physics-based bidisperse gas-particle drag model that is capable of predicting the drag force in a freely evolving bidisperse gas-solid suspension for a wide range of parameters are discussed. This discussion highlights the need for further investigation into the bidisperse gas-particle drag model.

\section{Acknowledgments}

This work is partially supported by Department of Energy grant DEFC26-07NT43098 through the National Energy Technology Laboratory (NETL). We would also like to acknowledge the National Science Foundation for partial support from award CBET 1134500. 


\section{Appendix A. Statistical representation of governing equations for a gas-solid flow}

Statistical models of multiphase flow are widely used to describe averaged conservation equations of mass, momentum, and energy of carrier and dispersed phases due to the statistical variability in multiphase flows. The two principal statistical approaches are: (i) The random field approach in which both carrier and dispersed phases are represented as random fields in the Eulerian frame, (ii) The point process approach in which the dispersed phase is represented as a stochastic point process in the Lagrangian frame while the carrier phase is represented as a random field in the Eulerian frame. In this section, the first approach is briefly reviewed because it is widely used in CFD of industrial applications.

In the Eulerian-Eulerian (EE) two-fluid theory approach, it is assumed that the conservation equations of mass, momentum and energy are valid for the entire system. The fluid-phase and the dispersed-phase cannot coexist at the same space location in a given time. Thus, an indicator field is used to represent the existence of phase $\beta$ at $(\mathbf{x}, t)$, that is (Drew and Passman, 1998)

$$
I^{(\beta)}(\mathbf{x}, t)= \begin{cases}1 & \text { if } \mathbf{x} \text { is in phase } \beta \\ 0 & \text { if } \mathbf{x} \text { is not in phase } \beta,\end{cases}
$$

Multiplying the conservation equations with the indicator field $I^{(\beta)}$ and using the corresponding topological equation (Drew and Passman, 1998) followed by the density-weighted phasic averaging yields the EE representation of the fluid phase and the solid phase. The fluid-phase conservation equations of 
mass and momentum in an isothermal non-reacting flow are given by

$$
\frac{\partial}{\partial t}\left(\rho^{(f)} \phi^{(f)}\right)+\nabla \cdot\left(\rho^{(f)} \phi^{(f)}\langle\mathbf{u}\rangle^{(f)}\right)=0
$$

and

$$
\begin{aligned}
\frac{\partial}{\partial t}\left(\rho^{(f)} \phi^{(f)}\left\langle\mathbf{u}^{(f)}\right\rangle\right) & +\nabla \cdot\left(\rho^{(f)} \phi^{(f)}\left\langle\mathbf{u}^{(f)}\right\rangle\left\langle\mathbf{u}^{(f)}\right\rangle\right)= \\
& -\phi^{(f)}\langle\mathbf{g}\rangle-\nabla \cdot\left(\rho^{(f)}\left\langle I^{(f)} \mathbf{u}^{\prime \prime(f)} \mathbf{u}^{\prime \prime(f)}\right\rangle\right) \\
& +\nabla \cdot\left\langle I^{(f)} \boldsymbol{\tau}\right\rangle-\sum_{\alpha=1}^{N_{c}}\left\langle\boldsymbol{\tau} \cdot \mathbf{n}^{(\alpha)} \delta\left(\mathbf{x}-\mathbf{x}_{\alpha}^{(I)}\right)\right\rangle
\end{aligned}
$$

where $\phi^{(f)}=\left\langle I^{(f)}\right\rangle$ is the mean fluid volume fraction, $\left\langle\mathbf{u}^{(f)}\right\rangle$ is the mean fluid velocity, $\mathbf{u}^{\prime \prime(f)}=\mathbf{u}^{(f)}-\left\langle\mathbf{u}^{(f)}\right\rangle$ is the fluid-phase velocity fluctuation, $\langle\mathbf{g}\rangle$ is any body force, $\boldsymbol{\tau}$ is the stress tensor, $\mathbf{n}^{(\alpha)}$ is the normal vector pointing outward from particle surface belonging to $\alpha^{\text {th }}$ class into the fluid phase, and $\delta\left(\mathbf{x}-\mathbf{x}_{\alpha}^{(I)}\right)$ is a generalized delta function at the fluid-particle interface $\mathbf{x}_{\alpha}^{(I)}$, and $N_{c}$ is the number of particle classes. The last term represents the mean momentum transfer between the fluid-phase and the particle class $\alpha$, known as gas-particle drag.

The corresponding conservation equations of mass and momentum for the particle class $\alpha$ are respectively:

$$
\frac{\partial}{\partial t}\left(\rho^{(\alpha)} \phi^{(\alpha)}\right)+\nabla \cdot\left(\rho^{(\alpha)} \phi^{(\alpha)}\left\langle\mathbf{u}^{(\alpha)}\right\rangle\right)=0
$$


and

$$
\begin{aligned}
\frac{\partial}{\partial t}\left(\rho^{(\alpha)} \phi^{(\alpha)}\left\langle\mathbf{u}^{(\alpha)}\right\rangle\right) & +\nabla \cdot\left(\rho^{(\alpha)} \phi^{(\alpha)}\left\langle\mathbf{u}^{(\alpha)}\right\rangle\left\langle\mathbf{u}^{(\alpha)}\right\rangle\right)= \\
& -\phi^{(\alpha)}\langle\mathbf{g}\rangle-\nabla \cdot\left(\rho^{(\alpha)}\left\langle I^{(\alpha)} \mathbf{u}^{\prime \prime(\alpha)} \mathbf{u}^{\prime \prime(\alpha)}\right\rangle\right) \\
& +\nabla \cdot\left\langle\boldsymbol{\tau}^{(\alpha)}\right\rangle+\left\langle\boldsymbol{\tau} \cdot \mathbf{n}^{(\alpha)} \delta\left(\mathbf{x}-\mathbf{x}_{\alpha}^{(I)}\right)\right\rangle \\
& +\sum_{\substack{\beta=1 \\
\beta \neq \alpha}}^{N_{c}}\left\langle\mathbf{f}_{\text {coll }}^{(\beta \rightarrow \alpha)}\right\rangle,
\end{aligned}
$$

where the last term in the Eq. A.5 is the mean momentum transfer, known as particle-particle drag, due to collisions with particle from other classes.

\section{Appendix B. Formulation of PUReIBM for a homogeneous bidis- perse gas-solid suspension}

The IB force is non-zero only inside the particle to generate a fictitious flow. This fictitious flow imposes the no-slip and no-penetration boundary conditions at the particle surface which is represented by discrete points in spherical coordinates. The computation of IB forcing at the $(n+1)^{\text {th }}$ timestep is specified as (Tenneti et al., 2010; Mehrabadi et al., 2015)

$$
\mathbf{f}^{n+1}=\rho^{(f)} \frac{\mathbf{u}^{d}-\mathbf{u}^{n}}{\Delta t}+\rho^{(f)} \mathbf{S}^{n}+\mathbf{g}_{\mathrm{IBM}}^{n}-\mu^{(f)} \nabla^{2} \mathbf{u}^{n}+\rho^{(f)} \mathbf{A}_{f}
$$

where $\mathbf{u}^{d}$ is the desired velocity at a computational grid point inside the particle that is used to impose the appropriate boundary condition.

In PUReIBM any instantaneous field $Q(\mathbf{x}, t)$ is decomposed into a mean and a fluctuating part as $\langle Q\rangle_{\mathcal{V}}(t)+Q^{\prime}(\mathbf{x}, t)$, where the operator $\langle\cdot\rangle$ represent the ensemble averaging. In a homogeneous suspension, the mean quantity is independent of the spacial coordinate. Additionally, it is legitimate to 
replace the ensemble averaging with volume averaging (Tenneti et al., 2010, 2011; Mehrabadi et al., 2015) given by

$$
\langle Q\rangle_{\mathcal{V}}(t)=\frac{1}{V} \int_{\mathcal{V}} Q(\mathbf{x}, t) d \mathbf{v},
$$

where $V$ is the volume of the region $\mathcal{V}$. Applying the above volume averaging on the conservation equations of mass (Eq. 8) and momentum (Eq. 9) gives us the volume-averaged mass and momentum conservation equations. Because the volumetric means are independent of spatial location, the conservation equation of the mean mass is trivially satisfied. The mean momentum conservation equations is then obtained as

$$
\rho^{(f)} \frac{d\langle\mathbf{u}\rangle_{\mathcal{V}}}{d t}=-\left\langle\mathbf{g}_{\mathrm{IBM}}\right\rangle_{\mathcal{V}}+\langle\mathbf{f}\rangle_{\mathcal{V}}-\rho^{(f)} \mathbf{A}_{f}
$$

The mean IB forcing term $\langle\mathbf{f}\rangle$ is computed by volume-averaging the IB force specified in Eq. B.1 over the region $\mathcal{V}$.

The phasic averaged momentum equation of the fluid phase is obtained by multiplying the Eq. 9 with the fluid-phase indicator function $I^{(f)}(\mathbf{x}, t)$ followed by averaging over the fluid region $\mathcal{V}^{(f)}$. The fluid-phase mean momentum equation then reads as

$$
\frac{d}{d t}\left\langle\mathbf{u}^{(f)}\right\rangle=-\frac{1}{\rho^{(f)}}\left\langle\mathbf{g}_{\mathrm{IBM}}\right\rangle_{\mathcal{V}}-\frac{1}{\rho^{(f)}} \sum_{\alpha=1}^{N_{c}}\left\langle\mathbf{s}_{M}^{(\alpha)}\right\rangle-\mathbf{A}_{f},
$$

where

$$
\left\langle\mathbf{s}_{\mathrm{h}}^{(\alpha)}\right\rangle=\frac{1}{V} \sum_{i=1}^{N_{\alpha}} \underbrace{\int_{\partial \mathcal{V}(p)}\left(-\psi \mathbf{I}+\mu^{(f)} \nabla \mathbf{u}\right) \cdot \mathbf{n}^{(\alpha, i)} d A}_{\mathbf{S}_{\mathrm{h}}^{(\alpha, i)}},
$$

represents the sum of hydrodynamic forces acting on all particles in size class $\alpha$. In the above equations $N_{c}$ is the number of size classes, $N_{\alpha}$ is the number 
of particles in $\alpha^{\text {th }}$ size class, $\psi$ is the fluctuating pressure, $V$ is the total volume of the system, and $\mathbf{n}^{(\alpha, i)}$ is the normal vector on the $i^{\text {th }}$ particle of $\alpha^{\text {th }}$ size class.

The phasic mean velocity of particle size class $\alpha$ is determined by averaging the dynamic equation of motion given in Eq. 11 using $\left\langle\mathbf{u}^{(\alpha)}\right\rangle=$ $1 / N_{\alpha} \sum_{i=1}^{N_{\alpha}} \mathbf{V}^{(\alpha, i)}$. Thus, the evolution equation of the mean momentum for $\alpha^{\text {th }}$ particle size class is

$$
\frac{d}{d t}\left\langle\mathbf{u}^{(\alpha)}\right\rangle=-\frac{1}{\rho^{(\alpha)}}\left\langle\mathbf{g}_{\mathrm{IBM}}\right\rangle_{\mathcal{V}}+\frac{1}{\rho^{(\alpha)} \phi^{(\alpha)}}\left[\left\langle\mathbf{s}_{\mathrm{h}}^{(\alpha)}\right\rangle+\sum_{\substack{\beta=1 \\ \beta \neq \alpha}}^{N_{c}}\left\langle\mathbf{f}_{\mathrm{coll}}^{(\beta \rightarrow \alpha)}\right\rangle\right]-\mathbf{A}_{f},
$$

where $\rho^{(\alpha)}$ and $\phi^{(\alpha)}$ are respectively the mass density and solid-phase volume fraction of $\alpha^{\text {th }}$ size class, and $\left\langle\mathbf{f}_{\text {coll }}^{(\beta \rightarrow \alpha)}\right\rangle$ represents the contact force per unit volume acting on $i^{\text {th }}$ size class due to particle collisions from particles in the $\beta^{\text {th }}$ size class. Due to the conservation of momentum in particle collisions, the average of the collision term $\left\langle\mathbf{f}_{\text {coll }}^{(\alpha, j \rightarrow i)}\right\rangle$ in Eq. 11 is zero.

The phasic mean momentum equation (Eq. B.6) is used to derive the mass-weighted mean momentum of the solid phase. The mass-weighted velocity of the solid phase is defined as

$$
\left\langle\widetilde{\mathbf{u}^{(p)}}\right\rangle=\frac{\sum_{\alpha=1}^{N_{c}} \rho^{(\alpha)} \phi^{(\alpha)}\left\langle\mathbf{u}^{(\alpha)}\right\rangle}{\sum_{\alpha=1}^{N_{c}} \rho^{(\alpha)} \phi^{(\alpha)}} .
$$

The mass-weighted conservation equation of the solid phase is then revealed as

$$
\frac{d}{d t}\left\langle\widetilde{\mathbf{u}^{(p)}}\right\rangle=-\frac{\phi\left\langle\mathbf{g}_{\mathrm{IBM}}\right\rangle_{\mathcal{V}}}{\sum_{\alpha=1}^{N_{c}} \rho^{(\alpha)} \phi^{(\alpha)}}+\frac{\sum_{\alpha=1}^{N_{c}}\left\langle\mathbf{s}_{\mathrm{h}}^{(\alpha)}\right\rangle}{\sum_{\alpha=1}^{N_{c}} \rho^{(\alpha)} \phi^{(\alpha)}}-\mathbf{A}_{f},
$$

where $\phi$ is the total solid-phase volume fraction. Note that the collision term $\left\langle\mathbf{f}_{\text {coll }}^{(\beta \rightarrow \alpha)}\right\rangle$ in Eq. B.6 is zero due to the conservation of momentum in particle 
collisions among all classes. By assuming all size classes have the same mass density ratio $\rho^{(\alpha)}=\rho^{(p)}$, an expression for the mean pressure gradient is obtained by subtracting Eq. B.4 from Eq. B.8 that has the following form:

$$
\begin{aligned}
\frac{d}{d t}\left\langle\widetilde{\mathbf{u}^{(p)}}\right\rangle-\frac{d}{d t}\left\langle\mathbf{u}^{(f)}\right\rangle & =\left(\frac{1}{\rho^{(f)}}-\frac{1}{\rho^{(p)}}\right)\left\langle\mathbf{g}_{\mathrm{IBM}}\right\rangle_{\mathcal{V}} \\
& +\left(\frac{1}{\phi \rho^{(p)}}+\frac{1}{(1-\phi) \rho^{(f)}}\right) \sum_{\alpha=1}^{N_{c}}\left\langle\mathbf{s}_{M}^{(\alpha)}\right\rangle .
\end{aligned}
$$

The above equation is used to determine the mean pressure gradient corresponding to a pre-determined mean slip Reynolds number. In PUReIBM, the accelerating frame moves at the mean velocity of the particles. Therefore, the mean particle velocity $\left\langle\widetilde{\mathbf{u}^{(p)}}\right\rangle$ and the corresponding mean acceleration $d\left\langle\widetilde{\mathbf{u}^{(p)}}\right\rangle / d t$ is zero in this accelerating frame. Additionally, the mean acceleration of the fluid phase $d\left\langle\mathbf{u}^{(f)}\right\rangle / d t$ in Eq. B.9 is discretized as $\left(\left\langle\mathbf{u}^{(f)}\right\rangle^{d}-\left\langle\mathbf{u}^{(f)}\right\rangle\right) / \Delta t$ where $\left\langle\mathbf{u}^{(f)}\right\rangle^{d}$ denotes the desired fluid-phase mean velocity. This velocity is set such that the desired mean slip Reynolds number is satisfied. The mean slip Reynolds number is defined based on the mean slip velocity between the gas and the solid phase, i.e., $\langle\mathbf{W}\rangle=\left\langle\widetilde{\mathbf{u}^{(p)}}\right\rangle-\left\langle\mathbf{u}^{(f)}\right\rangle$, and the Sauter mean diameter $\langle d\rangle=\left(\sum_{\alpha=1}^{N_{c}} \phi^{(\alpha)} /\left(\phi d_{\alpha}\right)\right)^{-1}$ given by

$$
R e_{m}=\frac{(1-\phi)|\langle\mathbf{W}\rangle|}{\nu^{(f)}}\langle d\rangle
$$

where $\nu^{(f)}$ is the gas-phase viscosity. Once the mean pressure gradient is established, the reference frame acceleration is determined from Eq. B.8. The mean pressure gradient and the reference frame acceleration are then used to solve the mean momentum equation given in Eq. B.3.

On the other hand, the momentum conservation equation for the fluctuating pressure and velocities is obtained by subtracting the mean momentum 
equation (Eq.B.3) from the instantaneous momentum equation (Eq.9). Periodic boundary conditions are imposed on fluctuating variables due to the homogeneity of the flow. A pseudo-spectral method is used with CrankNicolson scheme for the viscous terms and an Adams-Bashforth scheme for

the convective terms. A fractional time-stepping method based on Kim and Moin's approach (Kim and Moin, 1985) is used to advance the fluctuating velocities in time. Further details of PUReIBM are also reported by Tenneti et al. (2010).

\section{References}

Abate, A. R., Durian, D. J., Sep 2006. Approach to jamming in an airfluidized granular bed. Phys. Rev. E 74, 031308.

Bagchi, P., Balachandar, S., 2002. Effect of free rotation on the motion of a solid sphere in linear shear flow at moderate re. Physics of Fluids 14 (8), 2719-2737.

Beetstra, R., van der Hoef, M. A., Kuipers, J. A. M., 2007. Drag force of intermediate Reynolds number flows past mono- and bidisperse arrays of spheres. AIChEJ 53, 489.

Bokkers, G. A., Annaland, M. V. S., Kuipers, J. A. M., 2004. Mixing and segregation in a bidisperse gas-solid fluidised bed: a numerical and experimental study. Powder Technology 140, 176-186.

Chen, S., Doolen, G. D., 1998. Lattice-Boltzmann method for fluid flows. Annual Review of Fluid Mechanics 30 (1), 329-364. 
Chew, J. W., Hays, R., Findlay, J. G., Karri, S. R., Knowlton, T. M., Cocco, R. A., Hrenya, C. M., 2011. Species segregation of binary mixtures and a continuous size distribution of group b particles in riser flow. Chemical Engineering Science 66 (20), 4595-4604.

Chew, J. W., Hrenya, C. M., 2011. Link between bubbling and segregation patterns in gas-fluidized beds with continuous size distributions. AIChE Journal 57 (11), 3003-3011.

Cundall, P. A., Strack, O. D. L., 1979. A discrete numerical model for granular assemblies. Geotechnique 29, 47-65.

Drew, D. A., Passman, S. L., 1998. Theory of Multicomponent Fluids. Applied Mathematical Sciences. Springer, New York.

Fan, R., Fox, R. O., 2008. Segregation in polydisperse fluidized beds: Validation of a multi-fluid model. Chemical Engineering Science 63 (1), 272 285.

Fan, R., Marchisio, D. L., Fox, R. O., 2004. Application of the direct quadrature method of moments to polydisperse gas-solid fluidized beds. Powder Technology 139 (1), 7 - 20.

Feng, Z. G., Michaelides, E. E., 2004. The immersed boundary-lattice Boltzmann method for solving fluid-particles interaction problems. Journal of Computational Physics 195 (2), 602-628.

Garg, R., Tenneti, S., Mohd-Yusof, J., Subramaniam, S., 2010. Direct numerical simulation of gas-solids flow based on the immersed boundary method. 
In: Pannala, S., Syamlal, M., O'Brien, T. J. (Eds.), Computational GasSolids Flows and Reacting Systems: Theory, Methods and Practice. IGI Global.

Garzó, V., Dufty, J. W., Hrenya, C. M., 2007. Enskog theory for polydisperse granular mixtures. I. Navier-Stokes order transport. Physical Review E (Statistical, Nonlinear, and Soft Matter Physics) 76 (3), 031303.

Goldschmidt, M. J. V., Link, J. M., Mellema, S., Kuipers, J. A. M., 2003. Digital image analysis measurements of bed expansion and segregation dynamics in dense gas-solid fluidized beds. Powder Technology 138, 135159.

Hill, R. J., Koch, D. L., Ladd, A. J. C., 2001a. The first effects of fluid inertia on flows in ordered and random arrays of spheres. J. Fluid Mech. $448,213-241$.

Hill, R. J., Koch, D. L., Ladd, A. J. C., 2001b. Moderate-Reynolds-number flows in ordered and random arrays of spheres. J. Fluid Mech. 448, 243278.

Hoffmann, A., Janssen, L., Prins, J., 1993. Particle segregation in fluidized binary mixtires. Chemical Engineering Science 48 (9), 1583-1592.

Holloway, W., Benyahia, S., Hrenya, C. M., Sundaresan, S., 2011. Meso-scale structures of bidisperse mixtures of particles fluidized by a gas. Chemical Engineering Science 66 (19), 4403-4420.

Holloway, W., Yin, X., Sundaresan, S., 2010. Fluid-particle drag in inertial polydisperse gas-solid suspensions. AIChEJ 56, 1995-2004. 
Johnson, A. A., Tezduyar, T. E., 1997. 3D simulation of fluid-particle interactions with the number of particles reaching 100. Computer Methods in Applied Mechanics and Engineering 145 (3-4), 301-321.

Kiger, K. T., Pan, C., 2000. PIV technique for the simultaneous measurement of dilute two-phase flows. Journal of Fluids Engineering 122 (4), 811-818.

Kim, J., Moin, P., 1985. Application of a fractional-step method to incompressible Navier-Stokes equations. Journal of Computational Physics 59, 308-323.

Kurose, R., Komori, S., 4 1999. Drag and lift forces on a rotating sphere in a linear shear flow. Journal of Fluid Mechanics 384, 183-206.

Lebowitz, J. L., 1964. Exact solution of generalized percus-yevick equation for a mixture of hard spheres. Physics Review 13, A895-A899.

Lee, S., Durst, F., 1982. On the motion of particles in turbulent duct flows. International Journal of Multiphase Flow 8 (2), 125-146.

Louge, M. Y., Mastorakos, E., Jenkins, J. T., 1991. The role of particle collisions in pneumatic transport. Journal of Fluid Mechanics 231, 345359.

Mehrabadi, M., Tenneti, S., Garg, R., Subramaniam, S., 5 2015. Pseudoturbulent gas-phase velocity fluctuations in homogeneous gas-solid flow: fixed particle assemblies and freely evolving suspensions. Journal of Fluid Mechanics 770, 210-246. 
Nienow, A., Naimer, N., Chiba, T., 1987. Studies of segregation/mixing in fluidized beds of different size particles. Chemical Engineering Communications 62 (1-6), 53-66.

Norouzi, H., Mostoufi, N., Sotudeh-Gharebagh, R., 2012. Effect of fines on segregation of binary mixtures in gas-solid fluidized beds. Powder Technology 225, 7-20.

Oakley, T. R., Loth, E., Adrian, R. J., 1997. A two-phase cinematic PIV method for bubbly flows. Journal of Fluids Engineering 119 (3), 707-712.

Peskin, C. S., 2002. The immersed boundary method. Acta Numerica 11, $479-517$.

Prosperetti, A., Oguz, H. N., 2001. Physalis: A new $o(\mathrm{~N})$ method for the numerical simulation of disperse systems: Potential flow of spheres. Journal of Computational Physics 167 (1), 196-216.

Reis, P. M., Ingale, R. A., Shattuck, M. D., Apr 2007. Caging dynamics in a granular fluid. Phys. Rev. Lett. 98, 188301.

Rogers, C. B., Eaton, J. K., 1991. The effect of small particles on fluid turbulence in a flat plate, turbulent boundary layer in air. Physics of Fluids $3(5), 928-937$.

Rong, L., Dong, K., Yu, A., 2013. Lattice-Boltzmann simulation of fluid flow through packed beds of uniform spheres: Effect of porosity. Chemical Engineering Science 99 (0), $44-58$. 
Rong, L., Dong, K., Yu, A., 2014. Lattice-Boltzmann simulation of fluid flow through packed beds of spheres: Effect of particle size distribution. Chemical Engineering Science 116, 508-523.

Sarkar, S., Kriebitzsch, S., van der Hoef, M., Kuipers, J., 2009. Gas-solid interaction force from direct numerical simulation (DNS) of binary systems with extreme diameter ratios. Particuology 7 (4), 233-237.

Sato, Y., Hishida, K., Maeda, M., 1996. Effect of dispersed phase on modification of turbulent flow in a wall jet. Journal of Fluids Engineering 118 (2), 307-315.

Schiller, L., Naumann, A., 1935. A drag coefficient correlation. V.D.I. Zeitung.

Subramaniam, S., Mehrabadi, M., Horwitz, J., Mani, A., December 2014. Developing improved lagrangian point particle models of gas-solid flow from particle-resolved direct numerical simulation. In: Studying Turbulence Using Numerical Simulation Databases-XV, Proceedings of the CTR 2014 Summer Program. Center for Turbulence Research, Stanford University, CA, pp. 5-14.

Sun, B., Tenneti, S., Subramaniam, S., 2015. Modeling average gassolid heat transfer using particle-resolved direct numerical simulation. International Journal of Heat and Mass Transfer 86, 898 - 913.

Syamlal, M., 1987. The paricle-particle drag term in a multiparticle model of fluidization. Tech. Rep. DOE/MC/21353-2373, NTIS/DE87006500, National Technical Information Service, Springfield, VA. 
Syamlal, M., O'Brien, T. J., 1987. A generalized drag correlation for multiparticle systems. Tech. rep., Morgantown Energy Technology Center DOE Report.

Syamlal, M., Rogers, W., O’Brien, T. J., 1993. MFIX Documentation: Theory Guide. Tech. Rep. DOE/METC-95/1013, NTIS/DE95000031, National Energy Technology Laboratory, Department of Energy, see also URL http://www.mfix.org.

Tang, Y., Peters, E. A. J. F., Kuipers, J. A. M., 2016. Direct numerical simulations of dynamic gas-solid suspensions. AIChE Journal 62 (6), 19581969.

Tenneti, S., Garg, R., Hrenya, C., Fox, R., Subramaniam, S., 2010. Direct numerical simulation of gas-solid suspensions at moderate Reynolds number: Quantifying the coupling between hydrodynamic forces and particle velocity fluctuations. Powder Technology 203 (1), 57-69.

Tenneti, S., Garg, R., Subramaniam, S., 2011. Drag law for monodisperse gas-solid systems using particle-resolved direct numerical simulation of flow past fixed assemblies of spheres. Intl. J. Multiphase Flow 37 (9), 1072 1092.

Tenneti, S., Mehrabadi, M., Subramaniam, S., 2 2016. Stochastic lagrangian model for hydrodynamic acceleration of inertial particles in gas-solid suspensions. Journal of Fluid Mechanics 788, 695-729.

van der Hoef, M., 2007. Erratum. AIChE Journal 53 (11), 3020-3020. 
van der Hoef, M., Beetstra, R., Kuipers, J., 4 2005. Lattice-Boltzmann simulations of low-Reynolds-number flow past mono- and bidisperse arrays of spheres: results for the permeability and drag force. Journal of Fluid Mechanics 528, 233-254.

Wu, S., Baeyens, J., 1998. Segregation by size difference in gas fluidized beds. Powder Technology 98 (2), 139-150.

Xu, Y., Subramaniam, S., 2010. Effect of particle clusters on carrier flow turbulence: A direct numerical simulation study. Flow, Turbulence and Combustion 85, 735-761.

Yin, X., Sundaresan, S., 2009. Fluid-particle drag in low-Reynolds-number polydisperse gas-solid suspensions. AIChEJ 55 (6), 1352-1368. 